\title{
Current Trends and Perspectives of Detection and Location for Buried Non-Metallic Pipelines
}

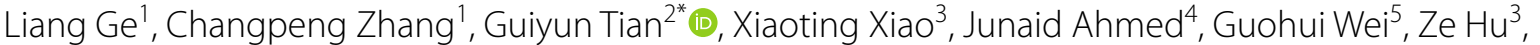 \\ Ju Xiang ${ }^{2}$ and Mark Robinson ${ }^{2}$
}

\begin{abstract}
Buried pipelines are an essential component of the urban infrastructure of modern cities. Traditional buried pipes are mainly made of metal materials. With the development of material science and technology in recent years, nonmetallic pipes, such as plastic pipes, ceramic pipes, and concrete pipes, are increasingly taking the place of pipes made from metal in various pipeline networks such as water supply, drainage, heat, industry, oil, and gas. The location technologies for the location of the buried metal pipeline have become mature, but detection and location technologies for the non-metallic pipelines are still developing. In this paper, current trends and future perspectives of detection and location of buried non-metallic pipelines are summarized. Initially, this paper reviews and analyzes electromagnetic induction technologies, electromagnetic wave technologies, and other physics-based technologies. It then focuses on acoustic detection and location technologies, and finally introduces emerging technologies. Then the technical characteristics of each detection and location method have been compared, with their strengths and weaknesses identified. The current trends and future perspectives of each buried non-metallic pipeline detection and location technology have also been defined. Finally, some suggestions for the future development of buried nonmetallic pipeline detection and location technologies are provided.
\end{abstract}

Keywords: Non-metallic pipeline, Pipeline detection and location, Non-destructive test and evaluation, Acoustic technologies

\section{Introduction}

Buried pipelines are important parts of urban infrastructure and are essential to the survival and development of the cities $[1,2]$. With the development of materials science and technology, the materials of urban buried pipelines are also changing [3]. In the past, a large number of metal pipes were used [4]. Currently, non-metallic pipes, such as plastic (polyethylene (PE) pipe, polyvinyl chloride (PVC) pipe, polypropylene (PP), etc.) pipes [5], ceramic pipes, concrete pipes, are becoming increasingly popular. Instead of metal pipes

\footnotetext{
*Correspondence: g.y.tian@ncl.ac.uk

2 School of Engineering, Newcastle University, Newcastle NE1 7RU, UK Full list of author information is available at the end of the article
}

in various pipe networks, such as water supply, drainage, heating, and oil and gas industry, non-metallic pipes have advantages of strong anti-pollution ability, lightweight, low cost, unsusceptibility to corrosion, easy construction and maintenance, etc. With the progress of society and the improvement of urban modernization, the construction technology of the buried pipeline is gradually becoming practical, economical, environmentally friendly, and secure. Trenchless technology has greatly promoted the construction and development of urban pipelines all over the world [6]. As for the detection and location technologies of the buried metal pipelines, non-destructive methods, such as electromagnetic detection, magnetic gradient method, artificial seismic method, and geological 
radar method, are frequently used. Vanaei [7], Karami [8], Hu [9], Feng [10], et al. carried out a specific analysis on the detection and location of the buried metallic pipelines. According to the analysis, the detection and location technologies of the buried metallic pipelines have become mature $[11,12]$. However, the detection and location of non-metallic pipelines still have many technical problems at present, because they are basically insulated and neither conductive nor magnetic [13]. Commonly used metal pipeline detectors cannot be used to detect and locate non-metallic pipelines [14]. The detection and location of non-metallic pipelines quickly, accurately, and conveniently is an urgent problem.

As illustrated in Figure 1, there are many potential detection and location methods for buried non-metallic pipelines. According to the technical characteristics and maturity, these methods can be divided into the following categories: electromagnetic induction technologies [15], electromagnetic wave technologies [16], acoustic technologies [17], other physics-based technologies [18], and emerging technologies [19].

The electromagnetic induction methods include the electromagnetic tracer line where the stimulation is placed on a metallic tracer line on the surface of the nonmetallic pipeline in order to produce the induction magnetic field. There is also, the tracer probe method which locates the position of the non-metallic pipe by the electromagnetic signal intensity generated by a probe placed in the pipe.

Electromagnetic wave technologies include groundpenetrating radar (GPR) using high frequency electromagnetic wave reflection imaging, and radio frequency identification (RFID) using electromagnetic wave communication.

Acoustic technologies include the pipe excitation method, elastic wave method, and point vibration measurement. The pipe excitation method applies sound at a specific frequency directly to the pipe, the receiver detects and locates the non-metallic pipeline based on the acoustic wave intensity received. The elastic wave method uses ground vibration exciters to generate elastic waves, and locate buried non-metallic pipelines according to the characteristics of the acoustic echo. Point vibration measurement is to apply vertical harmonic excitation at several points along the ground and locate buried non-metallic pipelines according to different resonances.

Other physics-based technologies based on changes in physical properties include infrared thermography, highdensity resistivity method (HDRM), inertial gyroscopes, electrical capacitance tomography (ECT), etc. Infrared thermography exhibits different fluid and ambient temperatures in non-metallic pipes. HDRM has different

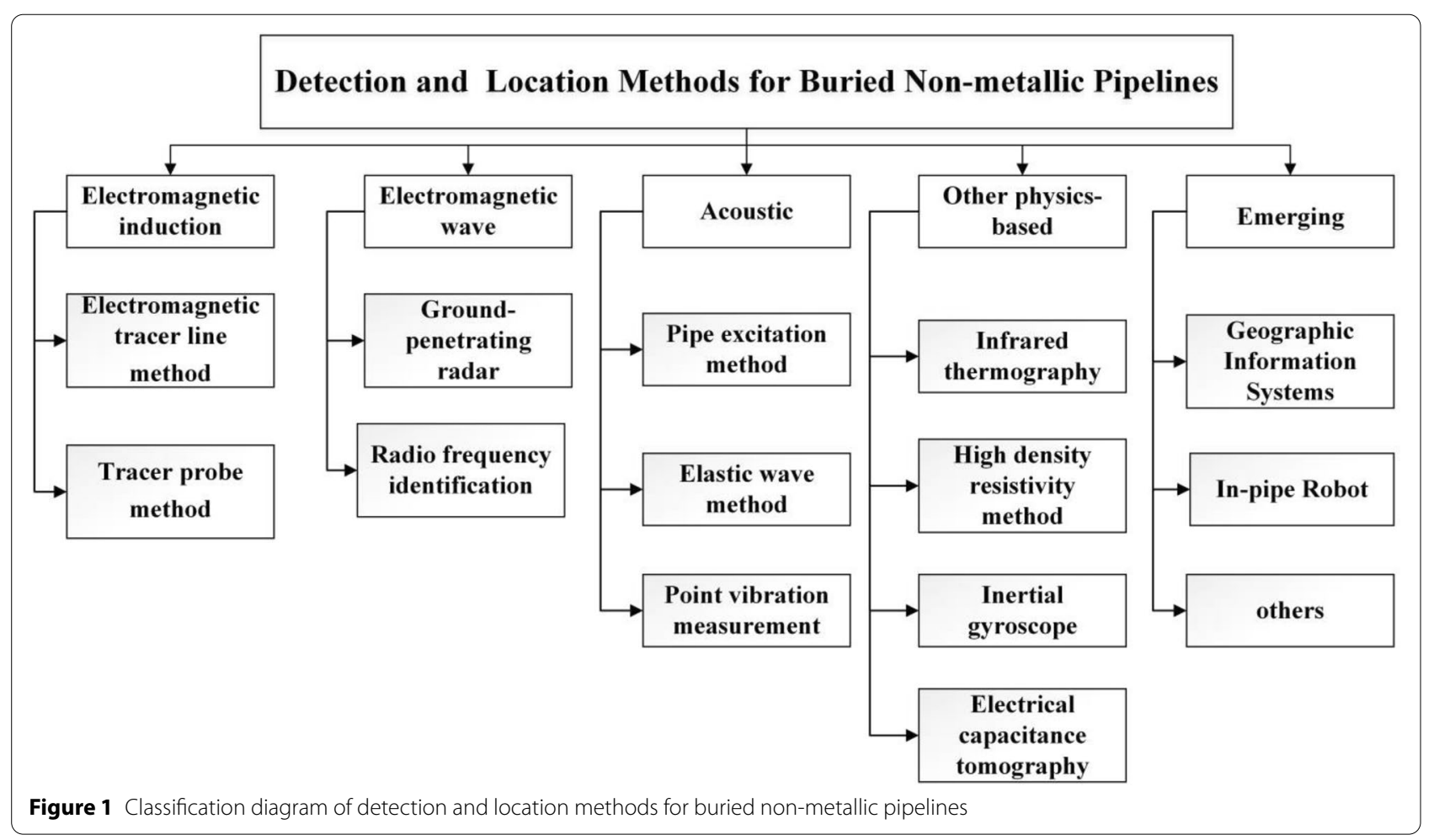


apparent earth resistivity. Inertial gyroscopes use angular momentum conservation to locate non-metallic pipes. ECT is a technique that uses a planar capacitance array to scan underground fault cavities, which can be used to detect and locate underground non-metallic pipelines.

Some emerging technologies based on new principles are attracting researcher's attention as well, such as geographic information system (GIS) and in-pipe robotbased inspection.

The objective of this paper is to provide state-of-theart of detection and location methods of non-metallic pipelines in various scenarios. The technical characteristics of the methods are compared so that the advantages and limitations of each technology are highlighted. The research trends and perspectives for new detection and location technologies of buried non-metallic pipelines are also discussed.

\section{Electromagnetic Induction Technologies}

The discovery of Faraday's law of electromagnetic induction lays a foundation for the use of electromagnetic induction technology to detect and locate pipelines [15]. Electromagnetic induction technology was first used to detect buried pipelines in 1910 [20]. In recent years, the detection and location of buried pipelines based on these technologies have been developed from metal pipelines to non-metallic pipelines [21].

\subsection{Electromagnetic Tracer Line Method}

The electromagnetic tracer line method follows the electromagnetic induction principle. During the detection, a metal wire with an obvious exposed point is laid on the surface of the non-metallic pipe as a tracer line. In this manner, a certain current can be conducted through the external transmitter [22]. The electromagnetic signal generated by the metal wire can be detected by the surface detectors and the location, buried depth, and direction of the non-metallic pipeline are determined as well.

According to the different principles of magnetic field generation in metal wires, the electromagnetic tracer line method can be divided into the direct connection method and induction method, as shown in Figures 2 and 3.

\subsubsection{Direct Connection Method}

Direct connection method [23, 24] uses the transmitter to apply a certain intensity of current to the tracer line and the position and depth of the non-metallic pipeline are determined by the magnetic field signal received by the ground detector.

The direct connection method [25] can adjust the emission current intensity at the transmitter end, which has a strong anti-interference ability. This method requires that
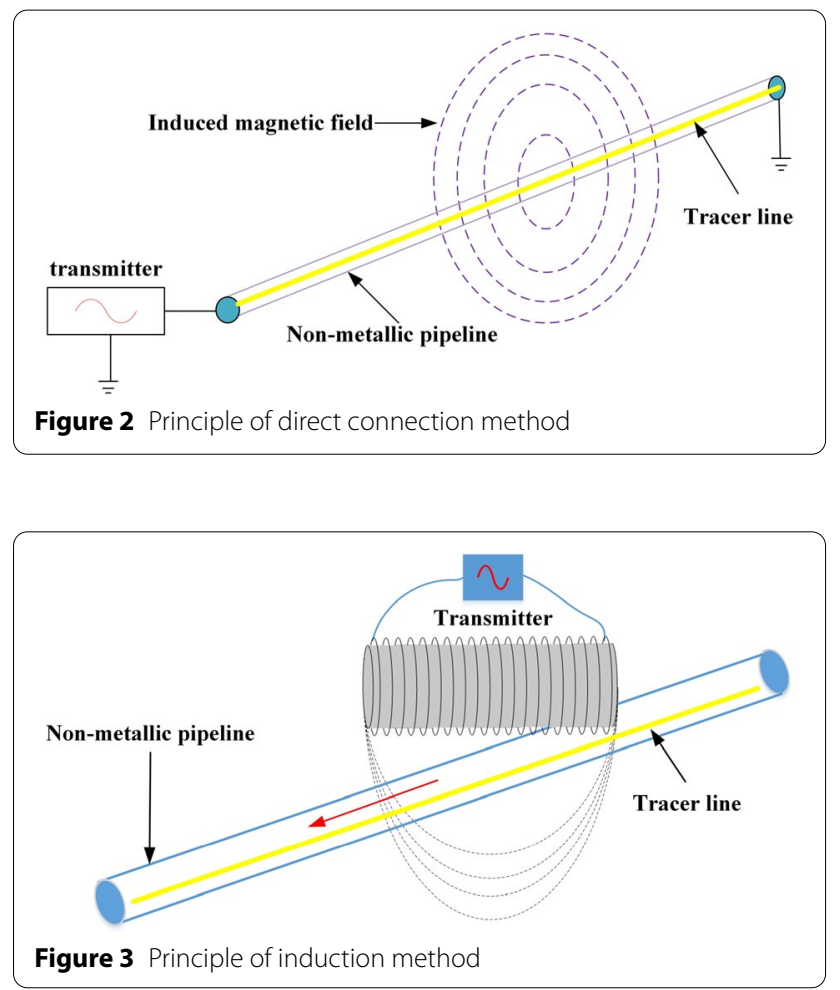

the tracer line must have an exposed point and have good grounding ability. It is mainly used in the location of a buried non-metallic pipeline with a complete tracer line. The detector should avoid the crossing of underground pipelines during the detection, so as to avoid other pipelines affecting the detection results.

\subsubsection{Induction Method}

The induction method [26, 27] uses the transmitter to emit an electromagnetic field, which makes the tracer line laid on the non-metallic pipeline generate a secondary electromagnetic field. Then, the position, depth, and direction of the buried non-metallic pipeline are determined by receivers according to the intensity of the electromagnetic field. The electromagnetic field generated by the transmitter must be a harmonic electromagnetic field. An example of the conductivity distribution at different frequencies is shown in Figure 4 [23].

According to whether the two ends of the transmitter need to be grounded or not, the induction method can be divided into electric dipole induction and magnetic dipole induction. The electric dipole induction is limited by the site conditions and the characteristics of the method itself. Therefore, it is rarely used in practice. The most commonly used method is the magnetic dipole induction method. It detects the buried nonmetallic pipeline by the generation of the secondary 


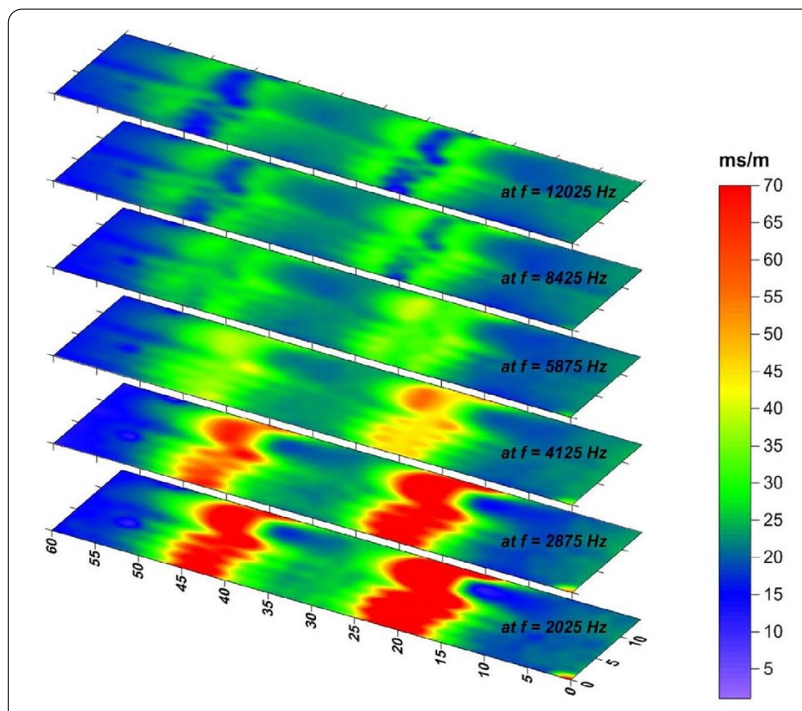

Figure 4 Image of conductivity distribution at different frequencies $(\mathrm{ms} / \mathrm{m})[23]$

electromagnetic field and receiving the electromagnetic abnormal signal of the pipeline [28].

Induction method is mainly used in the location of buried non-metallic pipelines with intact tracer lines. At present, the RD4000TL product [29] delivers accurate location of telecommunications infrastructure with the best performance, even in complex and electrically noisy environments [22].

The electromagnetic tracer line method is a proven method, which is easy for workers to operate. However, this method requires the installation of a tracer line, which needs to maintain good electrical connectivity and conductivity. When the tracer line breaks, this method will fail to work. In particular, the tracer line is easy to be destroyed while laying the pipeline or when the geological condition changes. The anti-interference ability of the location system is poor, and the magnetic field strength that can be detected for the deeply buried tracer line is weak [18]. To solve the above problems, the following aspects have been mainly studied and improved.

(1) The reliability of the tracer line has been improved. Many studies have addressed improvement in the tracer line, and the latest research proposed a copper-clad steel tracer line [30,31]. In these studies the tracer line is composed of three layers. The outermost layer is polyethylene (PE) plastic, which has the characteristics of protective insulation, anti-corrosion, and waterproof. The middle layer is copper, which can enhance the conductivity and enhance the strength of electric signal. The innermost layer is steel, which can enhance the strength of the line body and avoid the tracer line from being damaged during construction. The copper-clad steel tracer line solves the problems of insufficient tensile strength, poor aging resistance, waterproof joint, and external damage.

(2) The standard tracer line using the electromagnetic tracer line location method has been laid. The main influence factor of the signal current intensity in the tracer line is the loop resistance formed between the tracer line and the earth. The greater the loop resistance, the weaker the signal is, and vice versa. The loop resistance is closely related to the construction method of the tracer line, which directly affects the effectiveness and accuracy of the location. In view of the non-standard construction of the tracer line, many kinds of research have been carried out to formulate specifications. It put forward requirements for the laying position and buried conditions of the tracer line [32].

(3) The electromagnetic tracer line location technologies that are suitable for small signal-to-noise ratio (SNR) situations have been developed. This can be achieved by improving the transmission power and the tracer line material [30].

\subsection{Tracer Probe Method}

In the tracer probe method, a small transmitter is placed in a non-metallic pipeline [32], which is essentially equivalent to a magnetic dipole. The position and depth of the non-metallic pipeline can be detected by constantly changing the position of the transmitter in the pipeline, and the receiver is used on the surface to track the electromagnetic signal emitted by the transmitter. As shown in Figure 5, along the pipeline direction, the signal fluctuates several times before and after the pipeline probe. The receiving antenna on the surface with the same direction as the pipeline probe transmitting antenna will obtain the signal peak value above the pipeline probe. According to the different buried depths of the pipeline, a valley value will be obtained at a certain distance before and after the peak position. If the tracker detector is far away, the signal will rebound to a certain extent, reaching a relatively high value. The small peak value can be used to determine the location of buried non-metallic pipelines.

The tracer probe method emits an electromagnetic signal. By calculating the intensity of the electromagnetic signal and radio frequency of the signal detected and located, the location of the non-metallic pipeline can be determined. There are some mature products of the tracer probes, such as vLoc3-Cam Camera \& Sonde Locator [33] and Ritchie SR-20/8K/RD8000/remote Tracer Probe. In 2014, Chen [34] successfully used the 


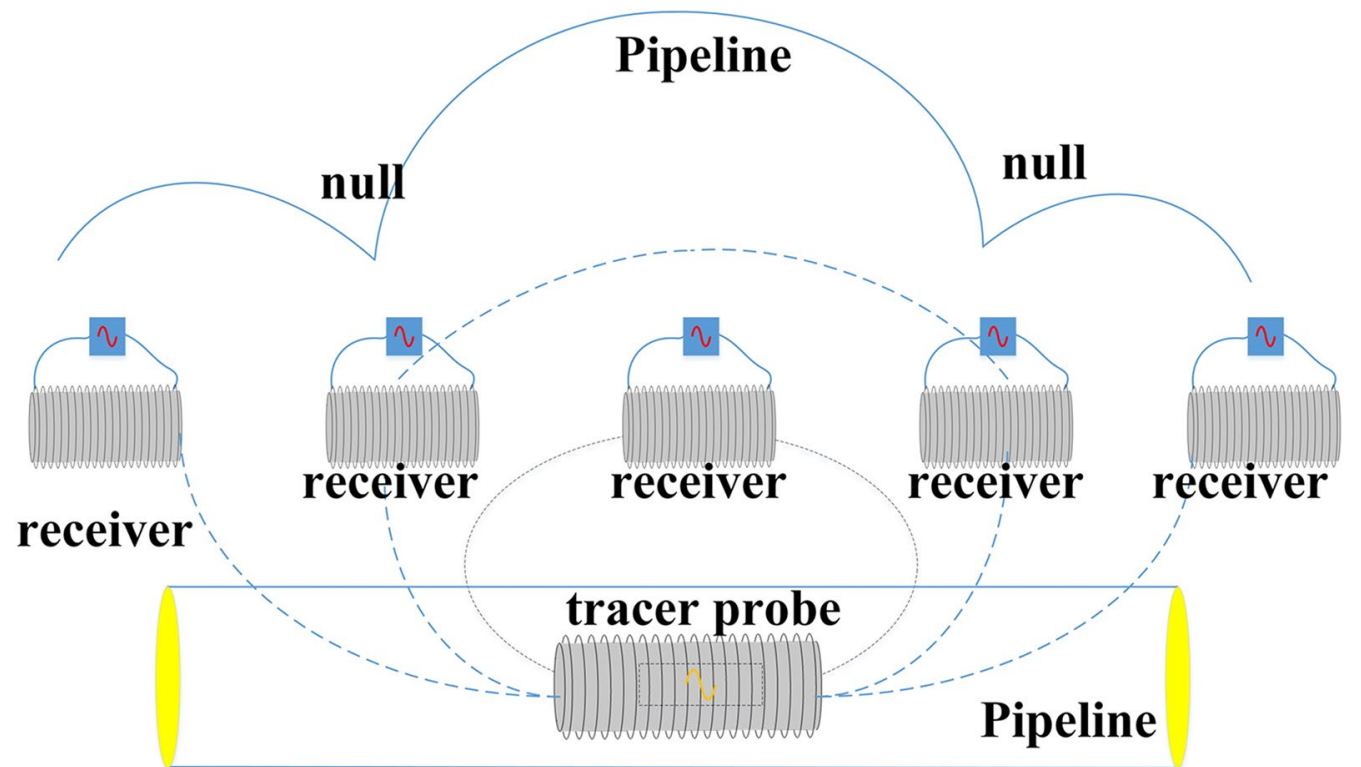

Figure 5 Configuration of tracer probe method

tracer probe method to locate the non-metallic pipeline in the complex areas where the pipes are densely laid, and the mutual interference is significant. The tracer probe method is relatively simple whereas the size of the probe, the robustness of the signal wire, and steering ability all have impacts on the location and detection of the nonmetallic pipeline [35]. Therefore, the improvements have been performed relating to the following aspects:

(1) The probe electromagnetic signal emission has been enhanced. The miniaturized power amplifier designed by Roshani et al. [36] has enhanced the emitting signals of the tracer probe without excessively increasing the probe size.

(2) The robustness of the wire and mobility of the probe has been enhanced. Miura et al. [37] designed a hollow conductive filament that can improve the toughness of the wire to detect and locate the farther pipeline path. Kim et al. [38] developed the magnetic flux leakage (MFL) system structure to reduce the friction on the pipeline wall. Stray et al. [39] designed the robotic carrier probe to move inside the pipeline and emit the electromagnetic signal.

Table 1 summarizes the advantages and disadvantages of electromagnetic induction detection and location technologies. The magnetic induction technologies detect and locate the buried non-metallic pipelines through the electromagnetic signal strength received by the surface receiver. Direct connection directly loads current to the tracer line, causing little interference to strong electromagnetic signals. The induction is easily disturbed by the magnetic field formed by the stray current from the nearby surface, and this reduces accuracy. The tracer probe method detects the electromagnetic signal emitted by the probe, and the receiver tracks the probe moving in the pipeline in real time, achieving high location accuracy.

\section{Electromagnetic Wave Technologies}

\subsection{Ground Penetrating Radar}

Ground Penetrating Radar (GPR) [16, 40] is a nondestructive geophysical location method that uses a high-frequency electromagnetic wave to detect the distribution of buried medium. Its working principle is to transmit the electromagnetic wave signal into the surface by using the transmitting antenna. When the electromagnetic wave [41] propagates into the surface and encounters targets with an electrical difference (such as holes, cracks), the electromagnetic wave will be reflected and received by the receiving antenna. The reflected wave can be analyzed to determine the position of the non-metallic pipe [3].

The working principle of GPR is shown in Figure 6. Figure 7 shows an actual GPR system. The propagation time $(\Delta t)$ from the transmitter to the receiver is given in Eq. (1):

$$
\Delta t=\sqrt{4 Z^{2}+X^{2}} / V,
$$

where $Z$ is the distance from the pipeline to the ground surface; $X$ is the distance between the transmitter and the 


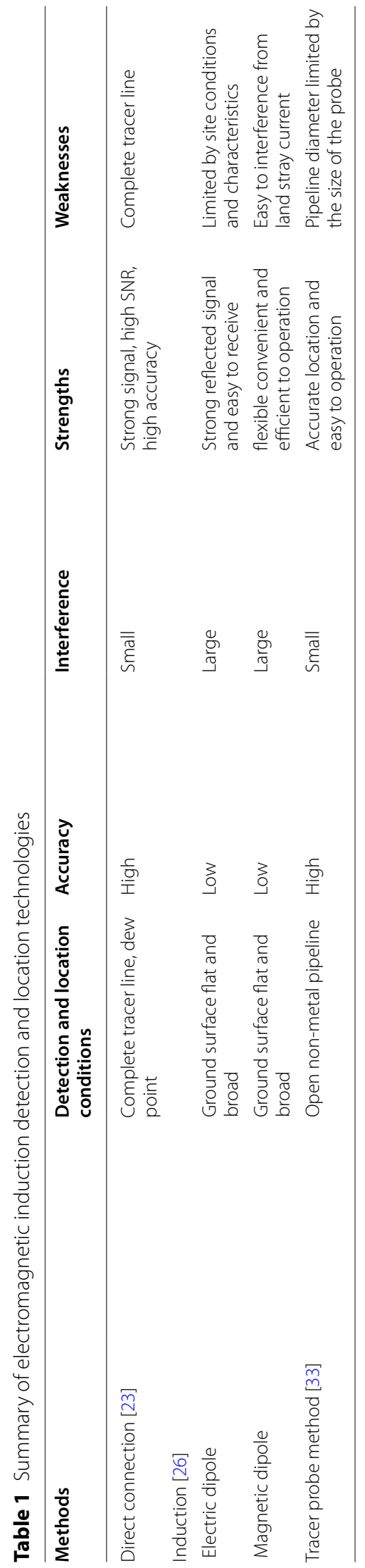



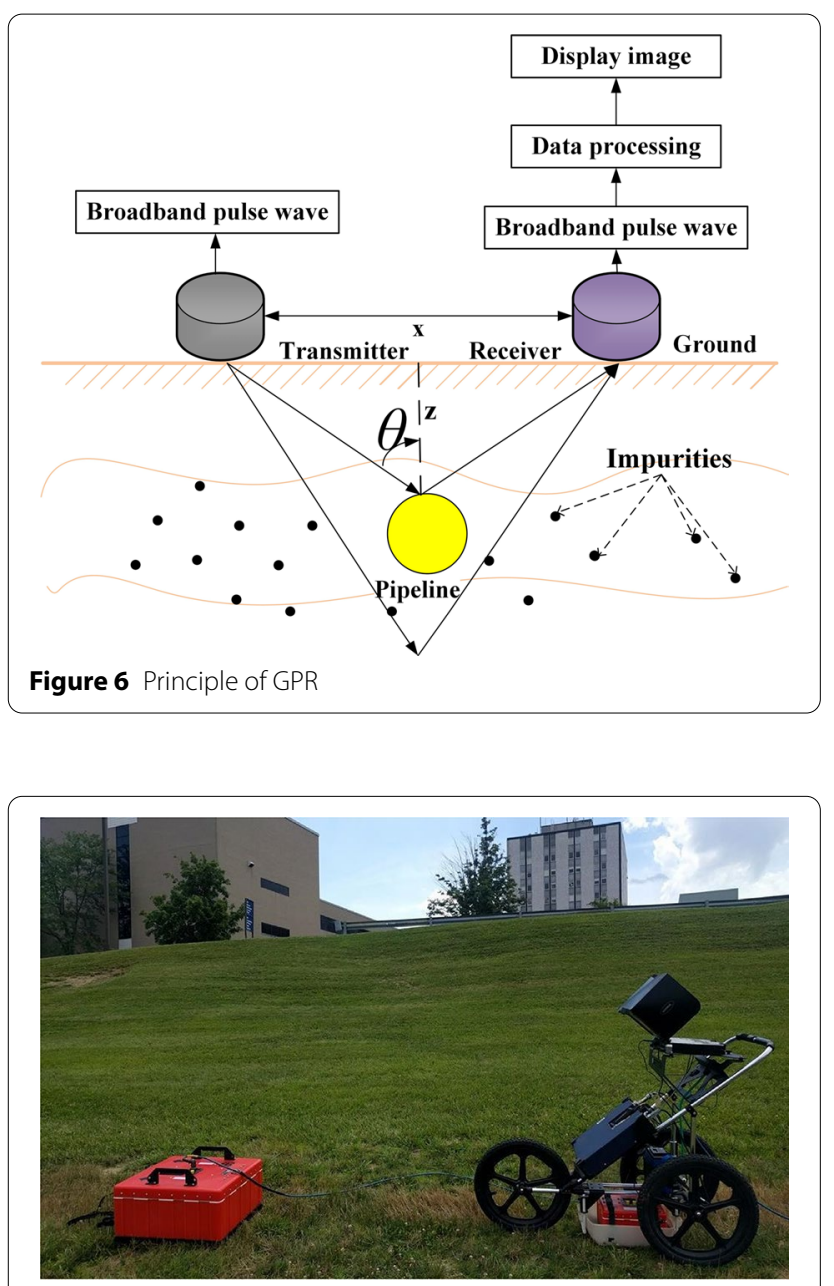

Figure 7 SIR-20 GPR system and radar antennae used for testing [42]

receiver, $V$ is the velocity of the electromagnetic wave in the soil.

The propagation speed of the electromagnetic wave is shown in Eq. (2):

$$
V=c / \sqrt{\varepsilon_{r}},
$$

where $\varepsilon_{r}$ is the relative permittivity of the medium, $c=3 \times 10^{8} \mathrm{~m} / \mathrm{s}$ is the velocity in freespace. When $X$ is assumed to be 0 , the depth $Z$ of the target can be calculated by:

$$
Z=V \cdot \Delta_{t} / 2
$$

The reflection coefficient of the electromagnetic wave signal encountering different media is shown in the following:

$$
R=\left(\sqrt{\varepsilon_{r 1}}-\sqrt{\varepsilon_{r 2}}\right) /\left(\sqrt{\varepsilon_{r 1}}+\sqrt{\varepsilon_{r 2}}\right),
$$

where $R$ is the reflection coefficient between the two media with the permittivities $\varepsilon_{r 1}$ and $\varepsilon_{r 2}$. It can be seen from Eq. (4) that the reflection coefficient of the electromagnetic wave mainly depends on the relative permittivities of the media on both sides of the reflection interface. When the contrast of the media increases, the reflection coefficient $R$ will also increase. Hence, the energy of the reflected wave will be enhanced. At the same time, the reflection coefficient is also related to the water content in the medium [43].

At present, the mature products of GPR on the market include RD1100 from Reddy, PulseEKKO PRO [41] from SSI, Detector DUO from IDS, SIR systems from GSSI, etc.

GPR method was initially used to detect underground cavities in highways to detect road quality [44]. Zeng et al. used the strong analytical power of high-frequency electromagnetic waves to detect buried metal pipelines [45]. Shaari et al. detected buried non-metallic pipelines [46], and Yigit et al. improved the GPR algorithm [47] for enhancing image resolution.

GPR has the advantages of high precision, high efficiency, and strong anti-interference ability. However, in the process of using GPR, it is necessary to calculate the existence, depth, and direction of buried pipelines based on parameters such as radar waveform, electromagnetic field strength, amplitude, and round-trip time. This has a high dependency on processors and algorithms. In addition, there are limitations when considering the detection and location of the buried pipelines with small diameters or located in saline-alkali soils and shale formations [48]. Moreover, the interpretations of B-scan images usually require professional experience and knowledge. In response to these problems, the following improvements and researches have been carried out.

(1) The data processing algorithm and the quality of the collected data have been improved. In the process of using the GPR, it has the disadvantages of image multiplicity and detection location limits in a complex environment. The Marching-Cross-Section (MCS) algorithm proposed by Dou et al. [49] can automatically and accurately locate the non-metallic pipeline under the investigated area with high recall and accuracy. Srinivas et al. [50] developed an algorithm to improve the image recognition of buried pipelines. Harkat et al. [51] designed a binary classifier with radial basis function (RBF) structure using high order statistic cumulant features (HOS), which improves the detection performance.

(2) The development of automatic adjustment of antenna transmission frequency to adapt to the depth of buried non-metallic pipelines has been 
made. The higher the center frequency of the surface penetrating radar, the higher vertical resolution, but the lower the accuracy of detection and positioning depth. Taking the vertical resolution and the detection and positioning depth into account, Kavi et al. [42] found that with the increasing depth of the research target, the image at 200 $\mathrm{MHz}$ is clearer than $400 \mathrm{MHz}$. The team is working on a surface-penetrating radar that changes the antenna transmission frequency in real time according to the depth of the buried pipeline.

\subsection{Radio Frequency Identification}

Thanks to the Unique Identification (UID) technology [52], the radio frequency identification (RFID) used for identification and tracking has developed rapidly. A complete RFID system [53] is composed of three parts: reader, electronic tag, and application software system. Its working principle is that the reader emits a radio wave signal at a specific frequency, and the tag is located near the buried non-metallic pipeline to receive the signal sent by the reader. After the energy is obtained by the induced current, the electromagnetic backscatter coupling, namely the principle of radar, sends the location and depth information stored in the tag chip (passive tag). Otherwise, the tag (active tag) actively sends a signal of a specified frequency. The reader reads and decodes this information, and then sends it to the central information system for relevant data processing and positioning of non-metallic pipes [54]. According to whether the tags need a power supply, RFID tags are divided into three categories: passive, semi-passive, and active $[55,56]$.

RFID mainly uses a tag to return information, but the tag is easy to be damaged and the signal return distance is significantly short. The deepest detection and location depth of the product on the market is $2 \mathrm{~m}$. Companies from China, such as Dahua, Yuanwang Gu and Chaowei, have mature products based on RFID. A diagram of the RFID monitoring system for structural health monitoring (SHM) is shown in Figure 8.

At present, RFID is mainly used for near-field communication of the Internet of things technology [57]. RFID chip-free sensor designed by Zarifi et al. [58] is used for real-time monitoring of pipeline integrity. Yclin et al. [59] used two-dimensional barcode and RFID technology to realize the location of objects and buried them beside a metallic pipeline to locate the pipeline.

RFID has the advantages of convenient early layout and easy operation. However, the tag is easily corroded over time, and the pipeline location depth is not deep [60, 61]. Currently, the main improvements and researches focus on the following aspects:

(1) The environmental adaptability of the beacon detector has been improved. Due to the fixed beacon is easy to be corroded and damaged, an anticorrosion shell material was developed. Zhang et al.

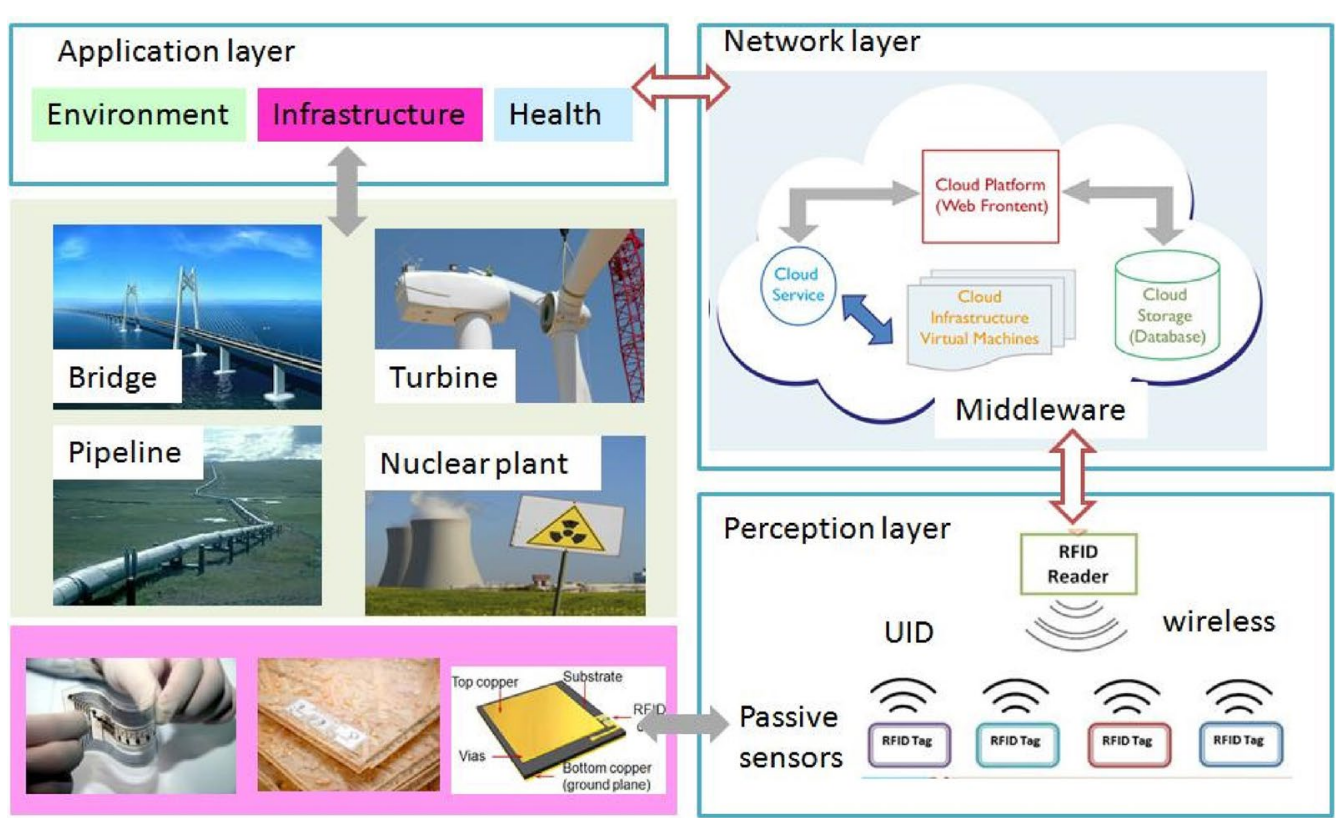

Figure 8 Passive RFID sensor networks for structural health monitoring (SHM) [53] 
[53] discussed emerging technologies that are used to implement passive antenna sensors and systems to make them more adaptable and reliable. They also conduct in-depth research in the UK to make RFID more capable of communication and location of deeper pipelines.

(2) The beacon location algorithm has been improved. For the problem of fixed beacon location distance, the anti-interference ability of the SEQ system designed by Vyas et al. [55] can be enhanced; the RSS algorithm proposed by Wu et al. [56] is based on the angle of linear regression and the advantages of differentially receiving a signal.

Table 2 summarizes the strengths and weaknesses of electromagnetic wave technologies for the detection and location of buried non-metallic pipelines. Electromagnetic wave technologies are used to transmit information. GPR analyzes the reflected high-frequency electromagnetic waves and locates buried pipelines with high accuracy and less susceptibility to interference. RFID uses the information transmitted by the electronic tag near the buried non-metallic pipeline to locate the buried pipeline with high accuracy and easy operation.

\section{Acoustic Technologies}

\subsection{Pipe Excitation Method}

The schematic diagram of the pipe excitation method approach is shown in Figure 9. This method is applicable to the case that the buried non-metallic pipeline can be touched from the surface (such as the surge tank) [17]. The basic principle of the pipe excitation method $[62,63]$ is to transmit acoustic signals into a buried non-metallic pipeline and then locate the pipeline by detecting the acoustic signal radiated from the pipeline to the surface through the detector on the surface $[64,65]$. The specific process of these technologies is as follows: Firstly, the recognizable acoustic signal is introduced into the buried pipeline. When the signal propagates forward along the pipeline, it will be transmitted to the surface through the soil. Then, the acoustic signal reaching the surface is detected by the detector on the surface. Finally, the position information of the pipeline is obtained by a certain calculation method. The outdoor experiment is shown in Figure 10.

To accurately locate the buried non-metallic pipeline by using the signal radiated from the pipeline, the amplitude, and phase information of the signal should be considered for processing. The approximate buried range of the non-metallic pipeline is preliminarily determined through the location area with the largest amplitude of the acoustic signal on the surface. Then the line is connected through the points with the same phase of the

Table 2 Summary of electromagnetic wave detection and location technologies

\begin{tabular}{llllll}
\hline Methods & Detection and location conditions & Accuracy & Interference & Strengths & Weaknesses \\
\hline GPR[16] & Big difference of electrical characteristic & High & Small & $\begin{array}{l}\text { High efficiency, anti-interference, } \\
\text { easy to operation }\end{array}$ & $\begin{array}{l}\text { Limitations by low diameter } \\
\text { and complex environment }\end{array}$ \\
RFID[53] & Buried tag near the buried pipeline & High & Small & Accurate location, easy to operation & Short life of the electronic tag
\end{tabular}

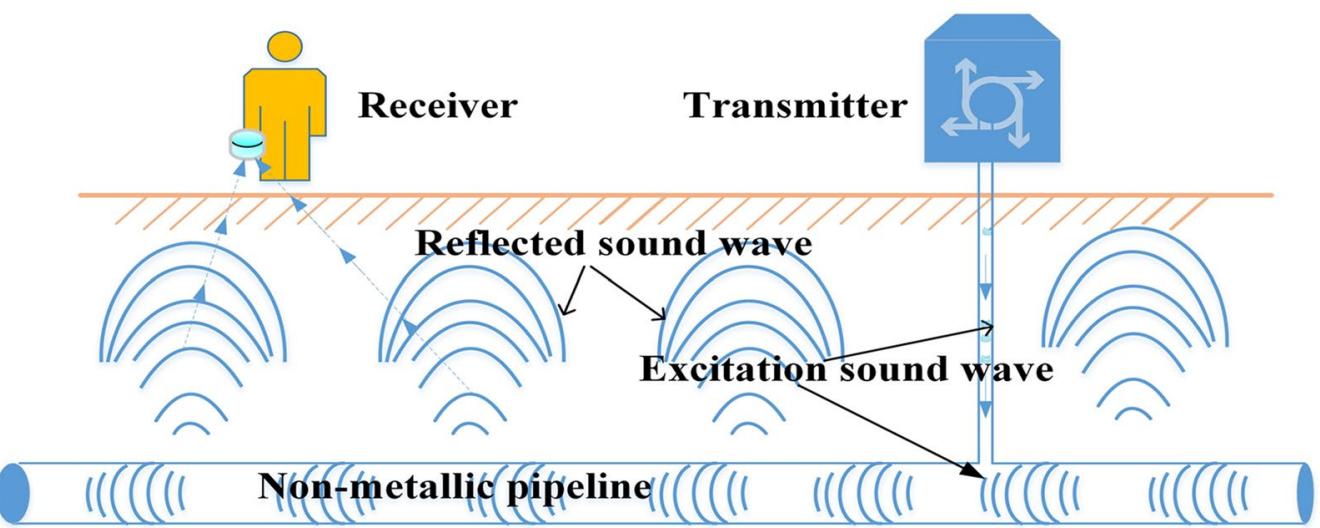

Figure 9 Principle of pipe excitation method 


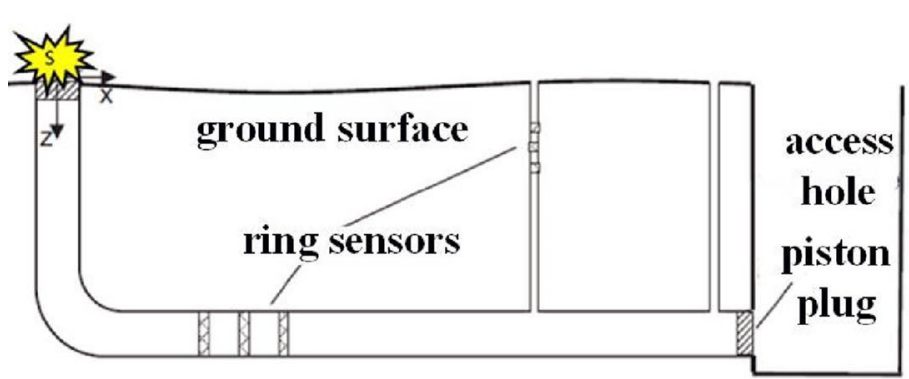

a

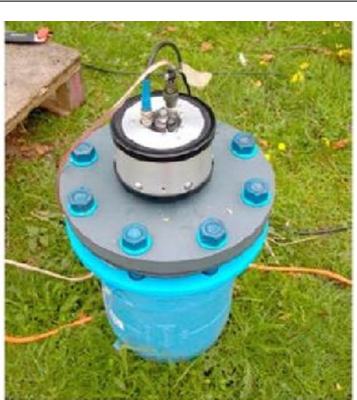

b

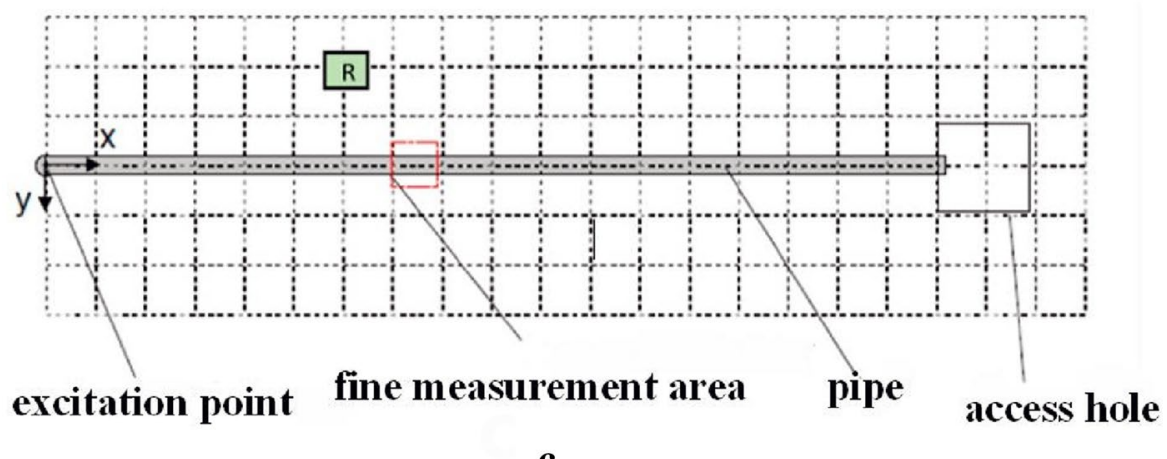

c

Figure 10 Experimental set-up of pipe excitation method: a pipe rig layout, $\mathbf{b}$ shaker mounted on plastic end-late, $\mathbf{c}$ grid of measurement points [67]

acoustic signal through which the pipeline direction can be further determined. The excitation signal frequency ranges from $10 \mathrm{~Hz}$ to $100 \mathrm{~Hz}$. In this range, the attenuation of the acoustic wave in the soil is the minimum. There are three ways to transmit sound waves into buried pipes, including active sound waves, passive sound waves, and resonant sound waves [66].

Active acoustic methods involve the introduction of sound waves on or in a pipe. It can be realized by connecting the sound source to an exposed point, such as a fire hydrant, or by inserting the noise source into the pipe. The resonance acoustic wave method is useful when the pipeline medium is non-compressed fluid (water in most cases), which can generate the detected sound wave in the pipeline by contacting the fluid surface and generating pressure waves. The research shows that the traditional sound transmission method can be used for the location of buried facilities. It can be regarded as an effective supplement to the existing technologies for locating metal and non-metal buried facilities as well [67].

At present, the products based on this principle include GPPL products of Guanchang Technologies Company, Gas Tracker jointly developed by MADE
Company and GDF French Gas Company, NPL-100 produced by Fuji in Japan, and System Combiphon produced by German Sewerin, etc.

The pipeline excitation method originated from water leakage detection $[68,69]$. The leakage noise propagates along the pipeline and spreads to the ground through the soil. On the ground, it can be absorbed by the auditory rod [70], and then the leakage point can be determined. Katz et al. [71] designed and assembled a prototype pipe excitation measurement device. In the early stage, the acoustic positioning system only processes the amplitude information of the collected signal to interpret the detection results, and its performance is poor. In order to overcome this defect, Muggleton et al. [72] proposed to use the phase of ground vibration data to improve the detection accuracy.

This method needs to inject sound waves into the buried pipeline from the pressure regulating box. The propagation speed of the sound wave in non-metallic materials is slow, while the attenuation of the sound wave is high. So it is easy to be interfered by the environment $[17,62,68,69]$. In response to these problems, the following improvements and researches have been made: 
(1) The optimal excitation waveform of acoustic waves can be determined. According to the natural frequency characteristics of PE pipe with different pipe diameters, the complex waveform was modulated to the region near the natural frequency. The composite signal from the transmitter consumes less energy when passing through each section of the pipeline. In this case, the signal transmission distance could be longer. Muggleton et al. [73] from the United Kingdom determined the optimal waveform of acoustic excitation through project research.

(2) The propagation characteristics of sound waves in the pipeline have been studied. Sound wave attenuates slowly in a liquid-filled pipeline. Xu et al. [74] established a three-dimensional model of a buried liquid filled pipeline to study the location technologies of buried pipeline based on acoustic propagation characteristics; Muggleton et al. [75] studied axisymmetric wave in a plastic pipe filled with liquid and used it to detect the location of the buried non-metallic pipeline.

(3) The acoustic location theory has been studied. Gao et al. [76-79] performed detection and location at a single point and analyzed the phase of the obtained signal in the frequency domain. A sudden change of the signal phase was found. To solve this problem, the axisymmetric wave propagation model was established. The earth's surface displacement caused by the radiation wave of the pipeline filled with fluids is studied, which lays the foundation for the acoustic location technology of the buried pipeline.

(4) The SNR and location range are improved. Through the encoding and decoding of the complex wave, the interference of external signal and the same frequency disturbance have been effectively avoided. So the measurement results will not be affected by external environmental factors. The signal-to-noise ratio and location distance are improved. Rahaman et al. [80] used AlN and D33 as piezoelectric sensors, which have the characteristics of high signal-to-noise ratio, high sensitivity, low noise, and linearity; Arumbu et al. [81] established the secondorder quadratic model of acoustic Doppler velocimeter to measure the acoustic signal, which represents the continuous model of signal-to-noise ratio and determines the optimal acoustic receiving area.

(5) The non-contact pipe excitation approach to radiate the sound wave into the pipeline has been studied. Muggleton et al. [82, 83] studied the location of the root of a tree by using ground vibration measurement. The results show that the direction of the plastic pipe adjacent to the tree root can be seen in the contour map of the two-dimensional signal under low frequency. Therefore, the acoustic wave can be radiated into the pipeline by applying excitation to the tree or ground near the pipeline without directly contacting the pipeline. In addition, the research on the relationship between the surface vibration characteristics and the buried depth of the pipeline shows that the pipe excitation method can provide the buried depth information of the pipeline [76].

\subsection{Elastic Wave Method}

The elastic wave method [84] is also known as the seismic wave method, including the compression wave method, shear wave method, and surface wave method. Compression waves and shear waves in the elastic medium are collectively called body waves. When the depth of the buried object is more than several meters, the compression wave method or shear wave method can better locate the target. When the buried object is buried within tens of centimeters, the surface wave method can better locate the target. In principle, the compressed wave method and the shear wave method are similar in locating buried objects. According to the different excitation signals and signal processing, the compression wave method (shear wave method) can be divided into the time-domain superposition method and frequency domain cross-correlation method. The former uses the short-time pulse signal as the excitation signal of the vibration source, while the latter uses the linear frequency modulation signal as the excitation signal of the vibration source [85]. The only difference between the compression wave method and the shear wave method is that in the elastic medium, the wave velocity of the compression wave is greater than that of the shear wave. Therefore, when the excitation frequency of both signals is set to be equal, the wavelength of the shear wave is smaller than that of the compression wave. So, the location or location resolution of the shear wave method is better than that of the compression wave method [86]. The surface wave method was first used to detect shallow buried mines. In recent years, some scholars used it to locate buried obstacles [87, 88], intervals [89], and cavities under sidewalks [90, 91].

When the time-domain superposition method is used to locate the buried non-metallic pipeline, a short-time compression wave (shear wave) pulse is first transmitted to the surface through the transmitter. Any discontinuity or interface with mismatched acoustic impedance during the buried propagation of the compression wave (shear wave) will be emitted [92, 93]. The reflection coefficient of the smooth inner surface of the pipeline and the contact surface of the gas is $100 \%$. The reflection coefficient 
of the contact surface between soil/rock and the external surface of the pipeline is small. Then, some receivers arranged on the ground surface are used to receive the reflected waves and convert them into electrical signals for output. The processed data is stored in the register. Finally, the position information of pipelines is obtained by the time-domain superposition algorithm in the upper computer. When the frequency-domain cross-correlation function method is used to locate the buried non-metallic pipeline, the excitation signal is changed to the linear frequency modulation signal and the location algorithm in the upper computer. Besides, the propagation and acquisition process of the elastic wave reflection signal

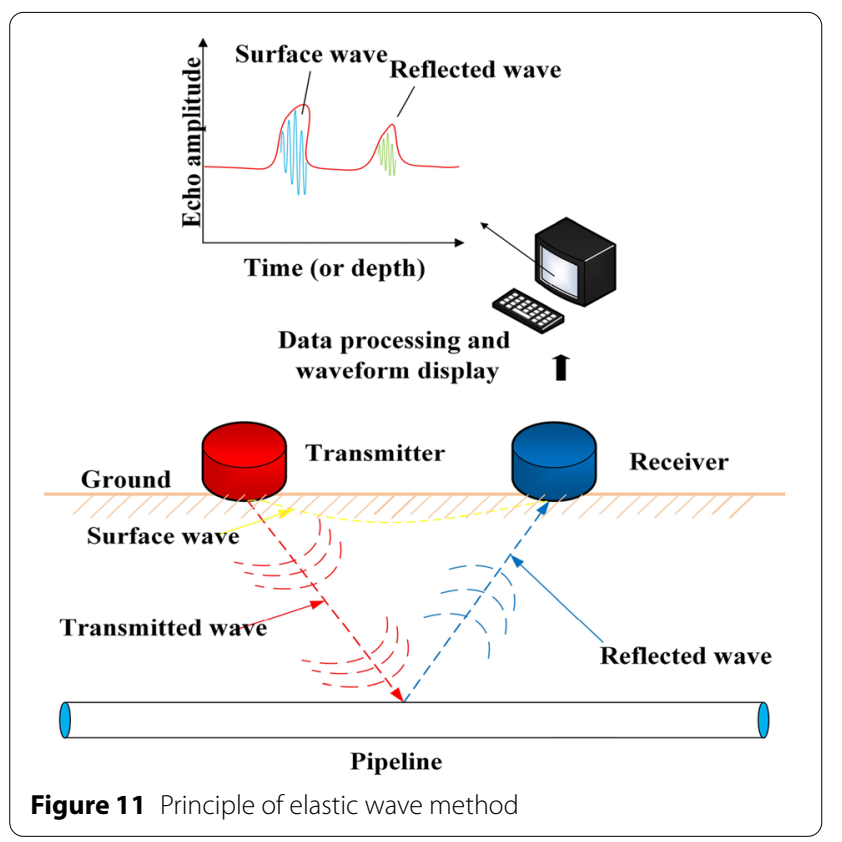

is the same as that of the time-domain superposition method.

Figure 11 is the schematic diagram of the principle of locating buried non-metallic pipelines by using the compression wave method or shear wave method. The amplitude and phase of elastic wave vibration signal reflected towards the surface are related to the reflection coefficient of the medium interface and the attenuation value of the elastic wave in the propagation medium. By analyzing the amplitude and phase of the output signal of the sensor, the impedance discontinuity in the soil below the location area, if there is, can be obtained. The reflected wave comes from the upper surface of the buried nonmetallic pipeline. So the location information of the buried pipeline is obtained [94, 95]. The compression wave experiment platform is shown in Figure 12.

When the general direction of the buried pipeline is known, several measuring lines perpendicular to the pipeline direction will be arranged on the surface, as shown in Figure 13. For an individual measuring line, firstly, several geophones are arranged along the measuring line, usually a velocity sensor or vibration sensor and a vibration source. Then, the excitation signal (short-time pulse signal or linear frequency modulation signal) is applied to make the vibration source radiate elastic wave. When the elastic wave propagates through the soil to the pipeline, it will be reflected by the surface of the pipeline, and finally, reach the ground surface. It is received by the geophone and converted to electrical signal output [96]. Finally, the position information of the pipeline on the measuring line is obtained by the delay information of the output signal of each detector. The same operation for the rest of the survey lines can then be done. Finally one can connect the pipeline mark points of each measured pipeline, and the line segment can be regarded as the actual

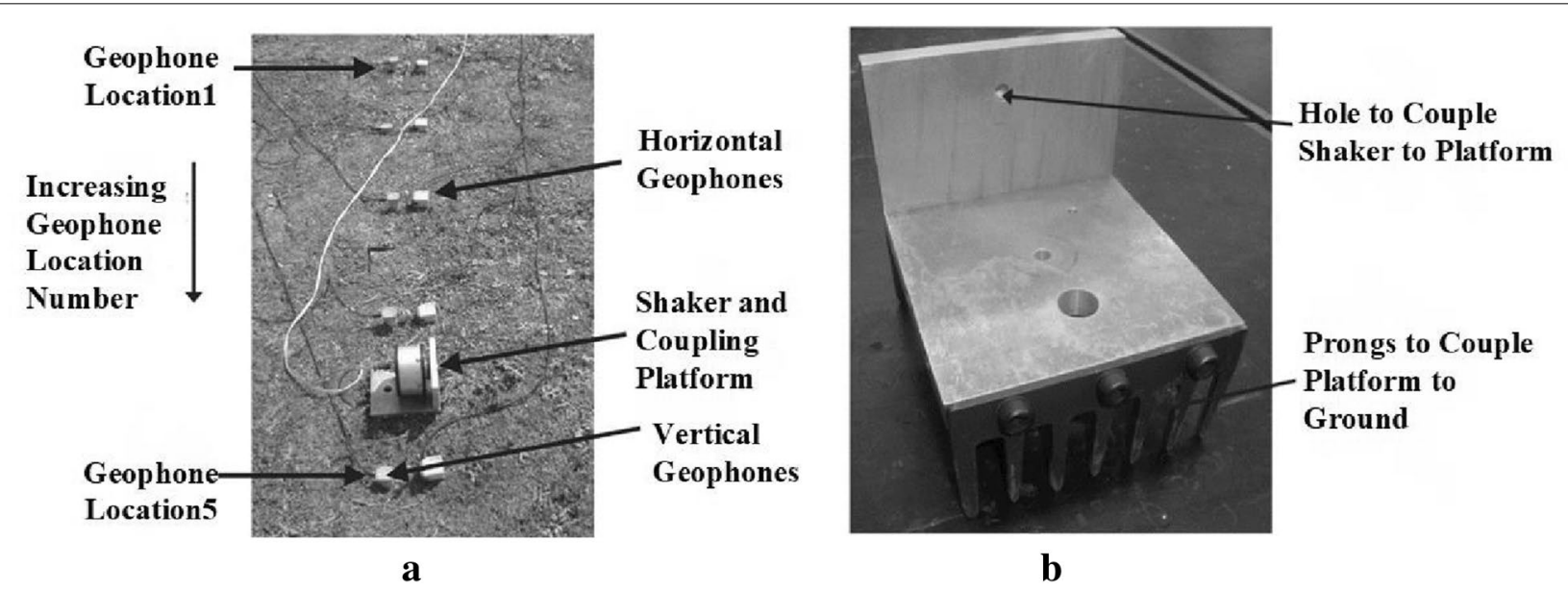

Figure 12 a Experimental set-up for compressional wave method, $\mathbf{b}$ specially designed platform used to couple the shaker to surface [86] 


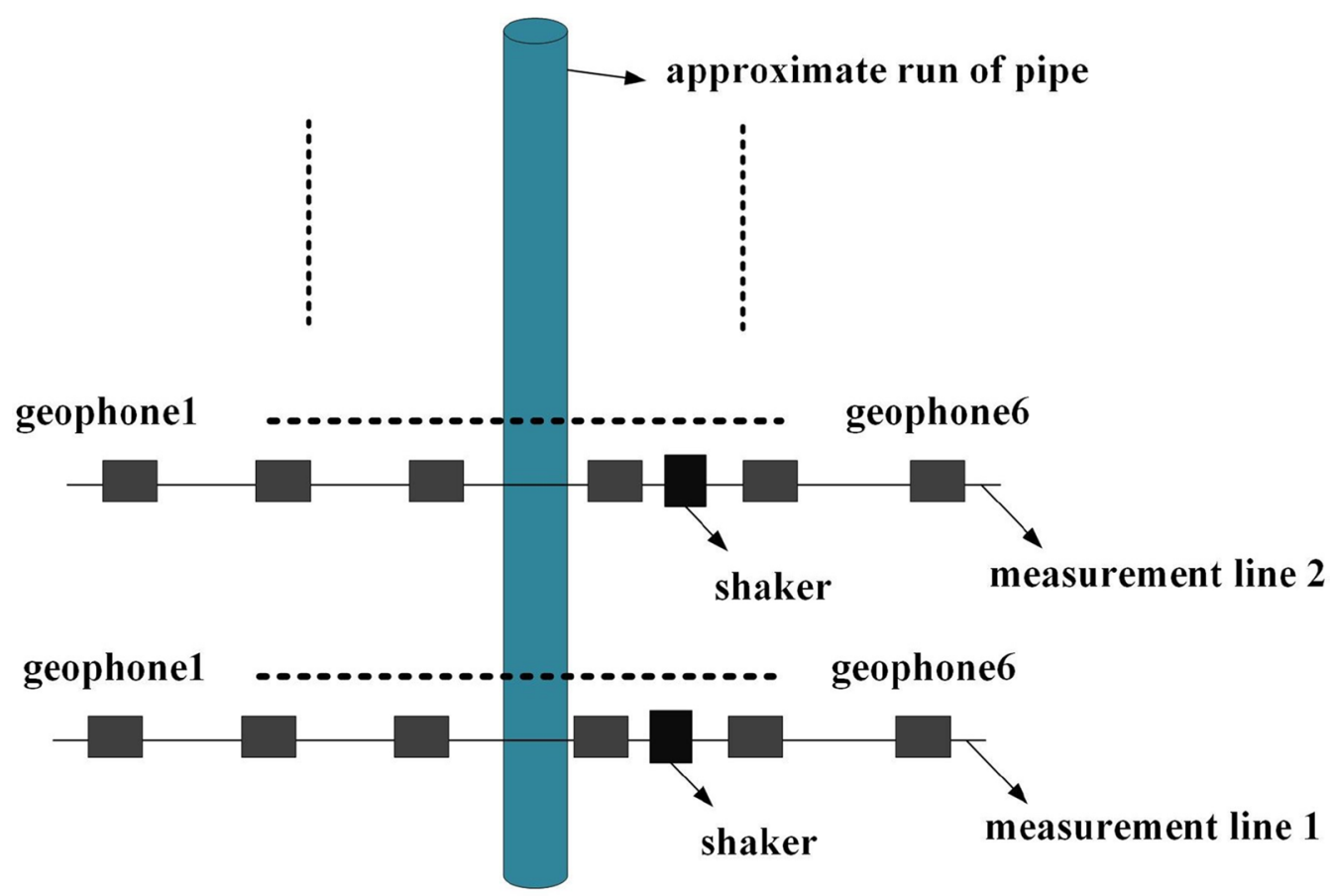

Figure 13 Sensor arrangement of elastic wave method

direction of the pipeline. The difficulty of this method lies in how to obtain the wave velocity. It is relatively easier to figure out the surface wave (elastic wave propagating along the earth's surface) and harder to obtain that of the volume wave velocity emitted into the soil.

Therefore, the in-situ measurement of the surface wave velocity is carried out at first. Then the volume wave velocity can be obtained according to the relationship between the velocities of the surface wave and volume wave.

The propagation distance of the compression wave (shear wave) from the source (shaker) $x_{s}$ to the number $i$ geophone $x_{s i}$ is shown in Eq. (5):

$$
d_{s i}=\left|x_{s}-x_{s i}\right| \text {. }
$$

The propagation time of the compression wave (shear wave) from the source to the number $i$ geophone is shown in Eq. (6):

$$
\tau_{s i}=\frac{d_{s i}}{c_{r}}
$$

where $c_{r}$ denotes the wave velocity. The distance between the compression wave (shear wave) propagating from the source to the pipeline and reflecting through the surface of the pipeline to the number $i$ geophone is shown in Eq. (7):

$$
d_{s p i}=\sqrt{\left(x_{s}-x_{p}\right)^{2}+y_{p}^{2}}+\sqrt{\left(x_{p}-x_{i}\right)^{2}+y_{p}^{2}},
$$

where $x_{p}, y_{p}$ represents the abscissa and ordinate of the endpoint of the pipe respectively. The propagation time of the compression wave (shear wave) propagating from the source to the pipeline and reflecting from the surface of the pipeline to reach the number $i$ geophone is shown in Eq. (8):

$$
\tau_{s p i \_s}=\frac{d_{s p i}}{c_{r} \frac{1+v}{0.87+1.12 v}},
$$

where $c_{r}$ is the velocity of the compression wave (shear wave), $v$ is Poisson's ratio of soil. It can be seen from Eqs. (5-8) that the time domain superposition method uses the time information of elastic wave signal reflected towards the surface to locate the pipeline.

The key equation of frequency domain cross-correlation function in Eq. (9):

$$
S_{x y}(f)=X *(f) Y(f),
$$

where $X *(f)$ is the frequency domain complex conjugate signal of the excitation signal $x(t), Y(f)$ is the frequency domain signal corresponding to the detector output signal $y(t), S_{x y}(f)$ which is the cross-spectral density function of $X *(f)$ and $Y(f)$. From Eq. (10), the 
cross-correlation function $R_{x y}(\tau)$ of $x(t)$ and $y(t)$ can be obtained in Eq. (10):

$$
R_{x y}(\tau)=F\left\{S_{x y}(f)\right\}=\int_{-\infty}^{\infty} S_{x y}(f) e^{-j 2 \pi f \tau} d \tau,
$$

where the cross-correlation function $R_{x y}(\tau)$ of $x(t)$ and $y(t)$ is the Fourier transform of its corresponding crossspectral density function. When the maximum value is taken, the corresponding independent variable is the time delay between $x(t)$ and $y(t)$. Therefore, the frequency domain cross-correlation function method can obtain the time delay information of the geophone output signal through the cross-correlation function.

When the short-time pulse compression wave (shear wave) is used to locate the buried non-metallic pipeline, the test signals of the six geophones on the surface are shown in Figure 14. Among them, a-f is the test signal from detector 1 to detector 6 in turn (all signals are translated in the $Y$ direction). According to the above six signals, the imaging results of the location area are shown in Figure 15, in which the black circle represents the actual position of the pipeline.

Using the elastic wave method to locate buried nonmetallic pipelines can not only detect the general range of the pipeline but also study the influence of various parameters such as soil type and buried depth on the location accuracy.

The elastic wave method has been widely used in underground oil and gas exploration [97]. Since crude oil is stored in hundreds or even thousands of meters deep underground, the common depth point (CPD) stacking technology [98] is not suitable for detecting buried shallow objects. Therefore, experts and scholars from home and abroad have improved the elastic wave method to detect shallow non-metallic pipelines [99].

The surface receiving device receives the reflected sound wave from the pipeline to detect the position of

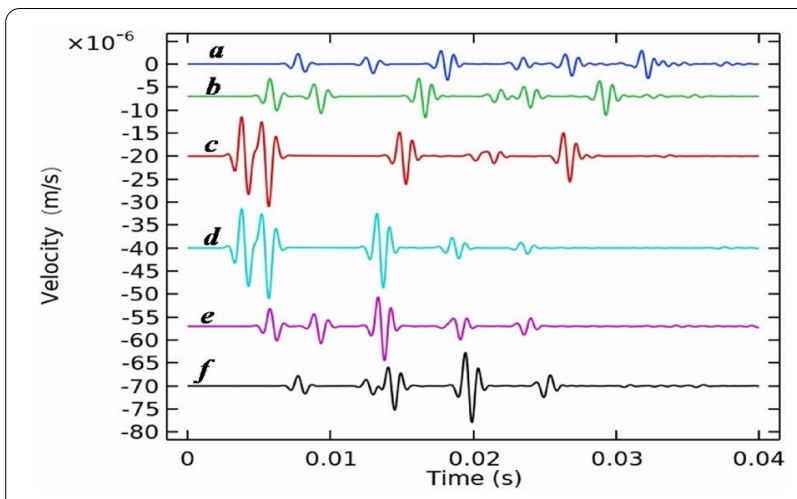

Figure 14 Test signals of geophone 1 to geophone 6

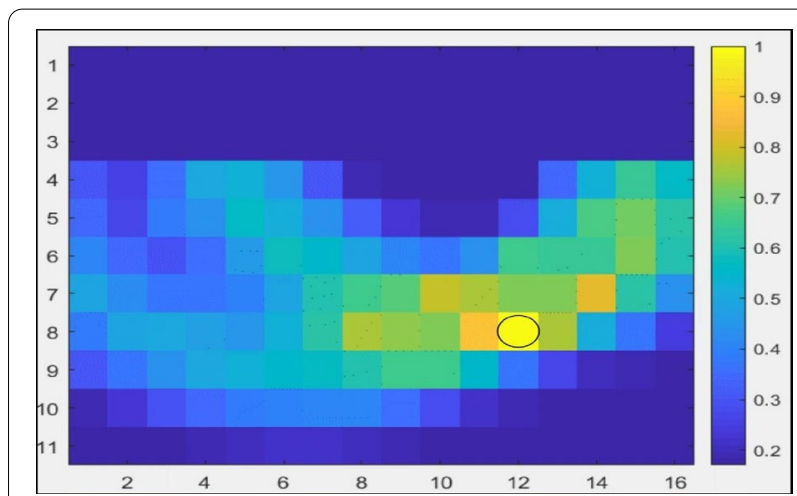

Figure 15 Two-dimensional image of the location area

the pipeline. The location result is easily affected by the buried cavity and other factors $[90,100]$. In order to solve the accuracy problem of the elastic wave method, the following aspects have been mainly improved and studied:

(1) The imaging algorithm has been studied. Sugimoto et al. [95] studied the algorithm for imaging the location area based on the elastic wave signal reflected from the buried. Papandreou et al. [85] proposed an imaging algorithm to calculate the time delay according to the cross-correlation function between the elastic wave signal and the excitation signal reflected by the buried pipeline. Then according to the time delay between the signals, the image of the shallow buried target detection can be seen clearly.

(2) The theory of elastic wave propagation has been studied. The method developed by Van Pamel et al. [93] was used to study the basic behavior of elastic wave scattering in terms of attenuation and dispersion. The finite element formula was established to solve the three-dimensional elastic dynamic scattering problem. Alber and Lo et al. [101, 102] studied the dispersion and attenuation characteristics of elastic waves in several typical porous soil media, such as clay, silt, sand, established the dispersion equation of elastic waves in the porous soil medium, and carried out numerical calculation and analysis.

(3) Array of multiple sensors has been made. The dual-probe multi-beam system designed by Xiong et al. [92] increases the reflected signal density and improves location accuracy.

(4) The excitation device of the elastic wave has been optimized. Papandreou et al. [84, 85] studied and designed a coupled vibration table. When the vibrator works, it is fixed on the vibration table, which 
can maximize the vibration energy concentration in the shear wave and improve the location accuracy.

\subsection{Point Vibration Measurement}

The point vibration measurement can be used to locate the shallow buried non-metallic pipelines. For this method, vertical harmonic excitation is applied at several points along the surface, and vibration (vibration/force) is measured at each point. When the excitation frequency is low, the surface behaves as a simple (mass-spring) system of a single degree of freedom [103] with a certain resonance frequency that depends on the density and elastic properties of the local soil.

The properties of the buried objects are different from those of the surrounding soil. The resonance frequency of the single degree of freedom system is related to the elastic properties of the buried objects. It will be changed compared to that without shallow buried objects. The establishment of the model is shown in Figure 16.

The ground surface can be regarded as a homogeneous elastic half-space, and its local static stiffness is $k$. When a vertical harmonic force is applied on a circular area with radius a, the local static stiffness $\mathrm{K}$ can be expressed in Eq. (11):

$$
k=\frac{f}{x}=\frac{2 E a}{1-v^{2}},
$$

where $E$ is the elastic modulus of the foundation, $v$ is the Poisson ratio of the foundation, and $x$ is the displacement.

The model in Figure 16 is simplified to a classical single degree of freedom system composed of mass, stiffness, and damping, as shown in Figure 17. The point vibration measurement device and measurement are shown in Figure 18. The motion equation of the system is given in Eq. (12):

$$
m \ddot{x}+c \dot{x}+k x=f,
$$

where $f$ is the applied force, $m$ is the mass, $k$ is the elastic stiffness, and $c$ is the damping, $\ddot{x}$ is the second-order

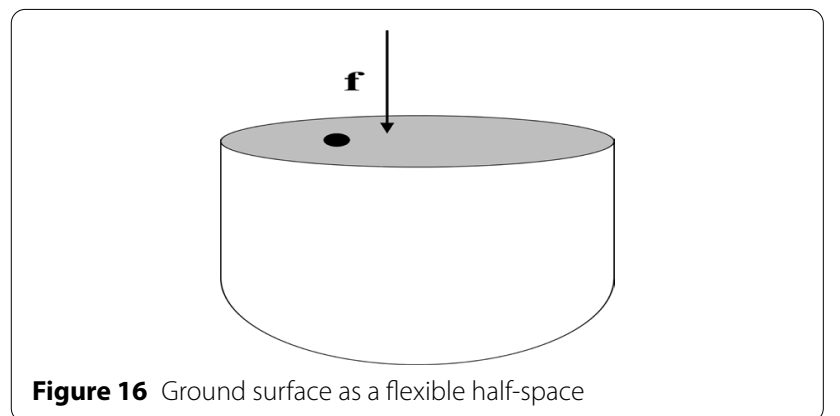

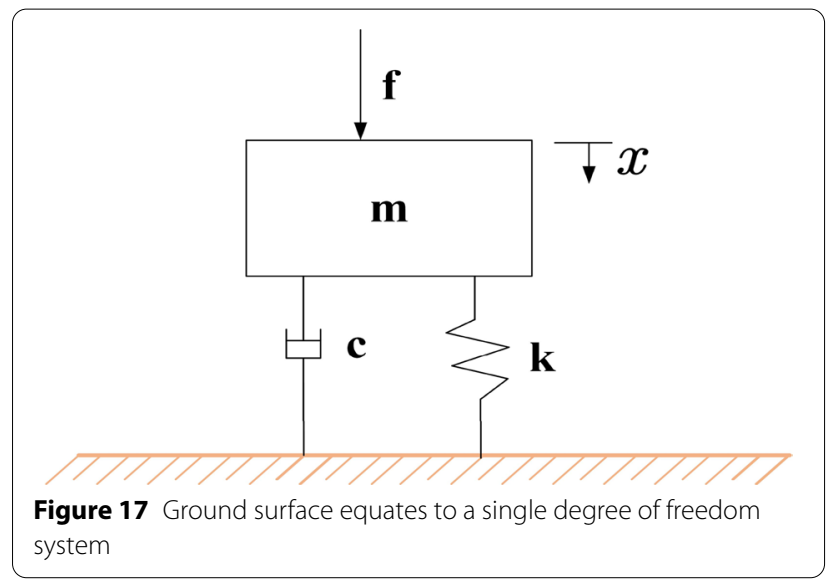

derivative of displacement to time, $\ddot{x}$ is the first-order derivative of displacement versus time.

$$
\begin{aligned}
& f=F e^{j \omega t}, \\
& x=X e^{j \omega t} .
\end{aligned}
$$

From Eq. (13) and Eq. (14), the point vibration (vibration/force) can be deduced, as shown in Eq. (15):

$$
\frac{\ddot{X}}{F}=\frac{1}{m-\frac{k}{\omega^{2}}-i \frac{c}{\omega}},
$$

where $m$ is the mass, $k$ is the spring stiffness, and $c$ is the damping, $\omega$ is the angular frequency.

Therefore, when there are shallow buried objects in the location area (about less than or equal to $30 \mathrm{~cm}$ ) [104, 105]. The buried object will affect the measurement stiffness of the ground surface, thus affecting the measured

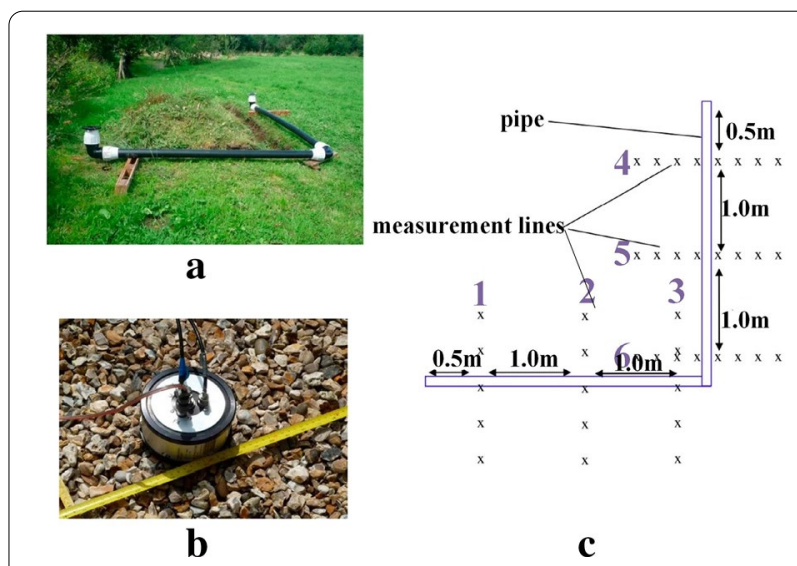

Figure 18 Point vibration measurements: a pipe rig before burial, b the acoustic source (shaker), $\mathbf{c}$ measurement lines (the cross are for illustrative purposes only) [103] 
resonance frequency. According to whether the resonance frequency of the measurement point changes, the presence of shallow buried objects below the point can be determined, and the location of non-metallic pipelines can be obtained. For the location of a simple buried nonmetallic pipeline, the point vibration method combined with the penetration judgment method can be used to preliminarily confirm the existence of the buried nonmetallic pipeline. The main advantage of point vibration measurement is that it can be carried out directly without the use of a sensor array, making it easy to use in the complex location area. In addition, the measurement results are easy to explain, and the location depth can be changed by increasing the excitation contact radius.

The point vibration measurement can detect the target by measuring the different resonance frequencies of a single degree of freedom system. This method is relatively fast in measurement and analysis but is not mature and the location depth is shallow [104, 105]. In order to solve the problem of the shallow depth in the location process of point vibration measurement, the following aspects have mainly been improved and studied at present:

(1) The friction stress at the pipe soil interface has been considered. Gao et al. [79] found that under the influence of soil load, the wave velocity across the tube soil interface will decrease.

(2) The contact with the surface has been increased to achieve a greater depth of location. Muggleton et al. [103] found that the location depth depends on the excitation contact radius. A greater location depth can be achieved by increasing the contact with the surface.

(3) Intelligent decisions have been made. Muggleton et al. [86] found that the development of multisensor and intelligent combinations can reduce the recognition of wrong targets and improve the location accuracy.
Table 3 summarizes the strengths and weaknesses of acoustic technologies of detection and location of buried non-metallic pipelines. The pipe excitation method is currently a commonly used method for acoustic detection and location of non-metallic pipelines. It directly generates sound waves by applying force on the pipeline to be detected. It has the advantages of high accuracy, low interference, and easiness to distinguish cross pipelines. The elastic wave method mainly uses the shear wave to excite the surface for generating sound waves. According to the intensity of the received reflected wave, the buried non-metallic pipeline is located. Point vibration measurement locates buried pipelines by measuring ground resonance, with shallow detection depth and low accuracy.

\section{Other Physics-Based Technologies \\ 5.1 Infrared Thermography}

Objects with a temperature above absolute zero degrees will radiate infrared rays due to their molecular motion $[18,106]$. The Infrared thermography method converts the power signal radiated by the object into an electrical signal through the infrared detector. By obtaining the thermal image corresponding to the heat distribution on the surface of the object, the location of the buried nonmetallic pipeline can be determined [107, 108].

The Infrared thermography method is mainly used for the location of buried heating non-metallic pipelines, such as oil, heating, etc. It can detect pipeline damage at the same time when detecting the pipeline position. The FLIR professional infrared thermal imager of the PUD Company has been widely used for research and engineering. An example of the Infrared thermal imaging is shown in Figure 19.

Shen et al. [109] detected the defects of the high-temperature pressure pipeline with infrared thermography. Bach et al [110] used infrared thermography to detect the water supply pipeline leakages. This technology has so far been used to locate and detect the heating pipe.

Table 3 Summary of acoustic detection and location technologies

\begin{tabular}{|c|c|c|c|c|c|}
\hline Methods & Location conditions & Accuracy & Interference & Strengths & Weaknesses \\
\hline Pipe excitation method [63] & Non-metallic pipes have a dew point & High & Small & $\begin{array}{l}\text { Easy to distinguish } \\
\text { the situation of } \\
\text { multiple pipelines } \\
\text { parallel }\end{array}$ & $\begin{array}{l}\text { The dew point is } \\
\text { needed, and the } \\
\text { gas needs to be } \\
\text { stopped }\end{array}$ \\
\hline Elastic wave method [85] & Any ground surface & High & Small & $\begin{array}{l}\text { Any ground surface, } \\
\text { the result is intuitive } \\
\text { and clear }\end{array}$ & $\begin{array}{l}\text { The operation is } \\
\text { complex and the } \\
\text { location efficiency } \\
\text { is low }\end{array}$ \\
\hline Point vibration measurement [104] & $\begin{array}{l}\text { Buried shallow enough non-metallic } \\
\text { pipeline }\end{array}$ & Low & Large & Easy operation & Shallow depth \\
\hline
\end{tabular}




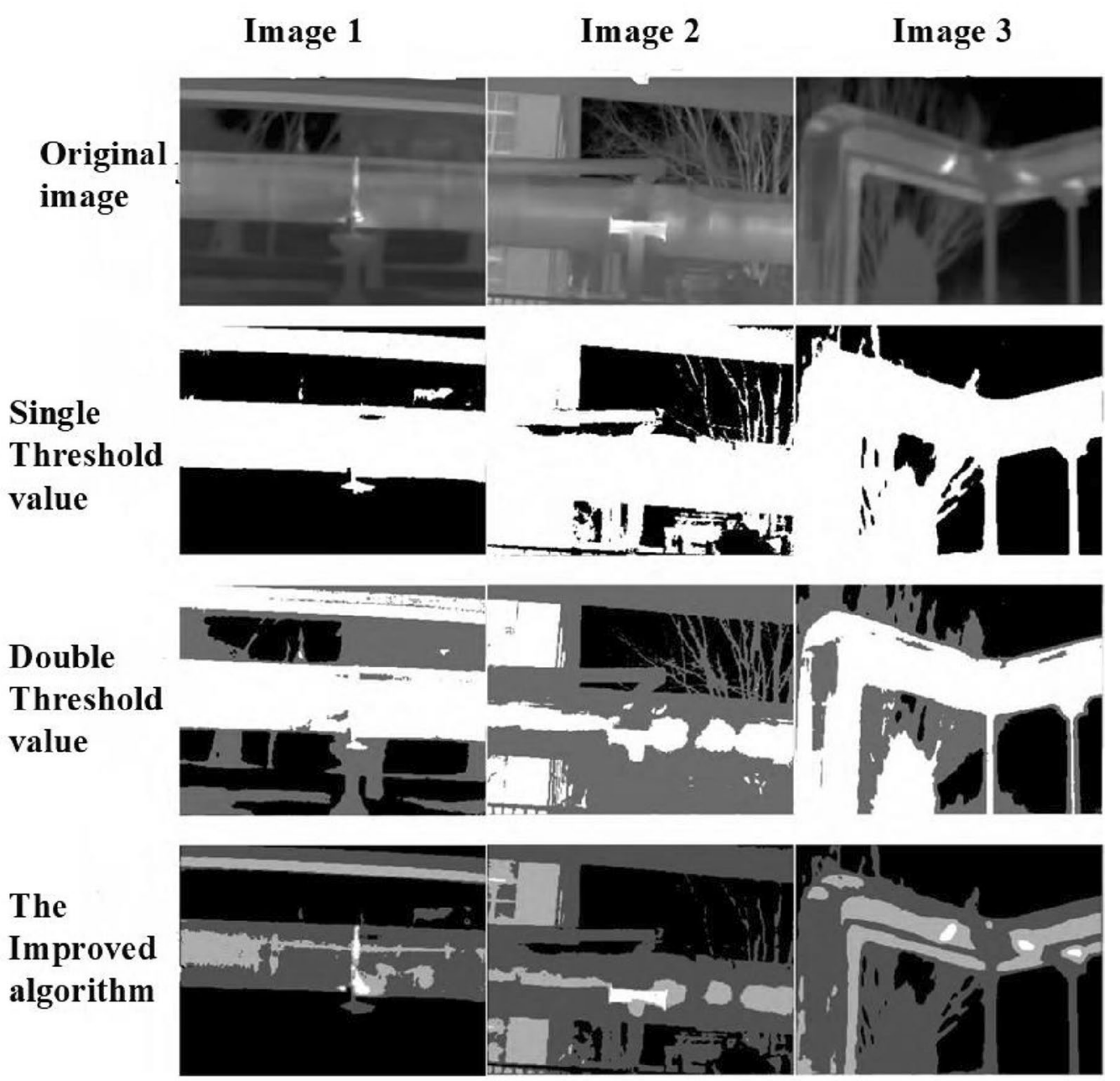

Figure 19 Segmentation images of three methods [18]

The target position can be obtained by analyzing the thermal image of the infrared radiometer whereas it is easy to be disturbed by nearby heat sources [106]. At present, the main improvement and conducted researches are as follows:

(1) A more advanced infrared thermal radiation algorithm to enhance the accuracy of the location has been developed. The improved method proposed by Shao et al. [18] can be applied to better distinguish the target pipeline and complex back surface.

(2) Combined with unmanned aerial vehicles (UAV) and other low altitude equipment, the location has been more convenient and faster. L Wang et al. [107] combined infrared location with UAV to carry out macroscopic location, saving time for the second stage detailed location.

\subsection{High Density Resistivity Method}

The high density resistivity method (HDRM) is a kind of direct current (DC) electrical prospecting method $[111,112]$. It is a geophysical method based on the electrical difference between the buried target and surrounding media [113], which is also known as Electrical resistivity tomography (ERT) [114-116]. In this method, the potential difference and power supply current are measured by different electrode arrangements, to obtain the apparent resistivity of the buried target and surrounding media. Due to the difference in electrode distance, the apparent resistivity of different depths can be obtained. According to the distribution law of apparent resistivity, the buried electrical property changes can be understood, and the measurement of the buried non-metallic pipe position is finally achieved. The principle and the device diagram of the resistivity method are shown in Figure 20. 


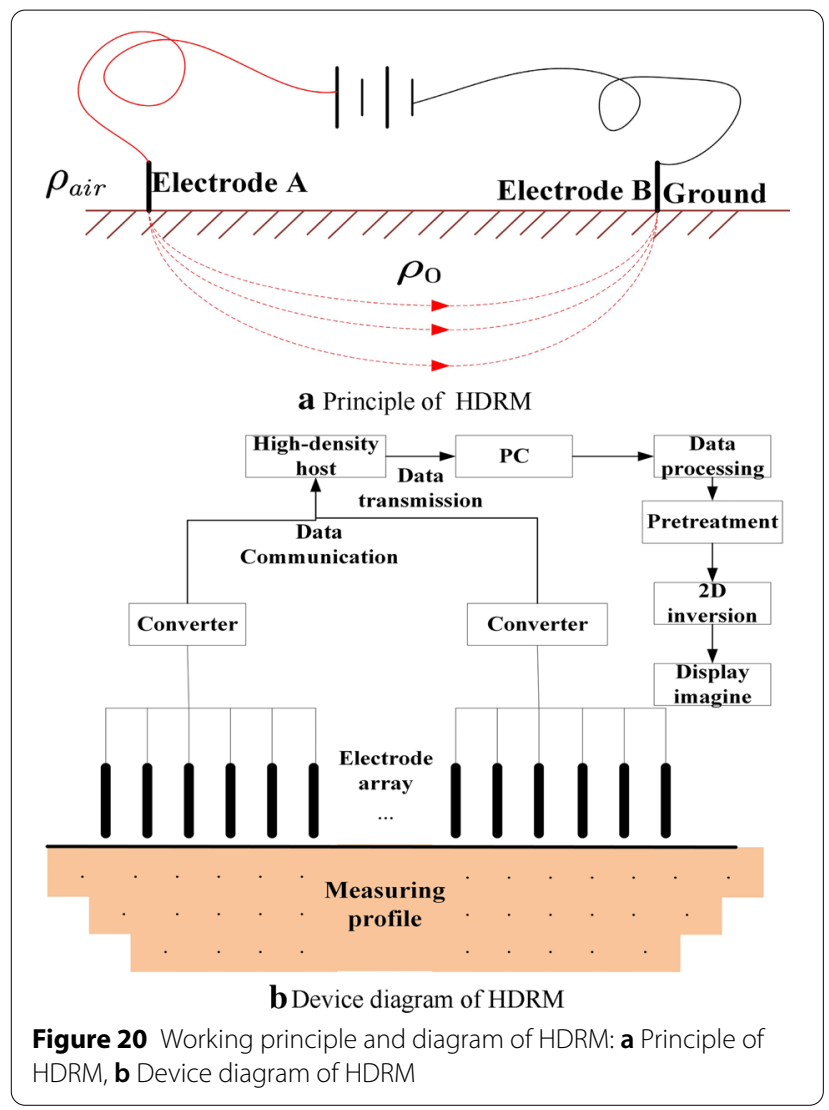

In Figure 20a, $\rho_{0}$ is the apparent resistivity between electrodes A and B; Figure 20b illustrates the diagram of HDRM. The two-dimensional section is shown in Figure 21.

Zhang et al. [117] used the high-density resistivity method to detect and locate the buried water supply network in 2009. Shi et al. [118] explored the pipeline under the earth dam in 2019 to prevent major damage by dam failure.

The high-density resistivity method is mainly used in the case that the buried soil is uniform without any impurity. According to the difference of resistivity, the location of the non-metallic pipeline can be obtained. At present, there are mature products such as DUK- 2 and HX-GMD01A. DUK-2 is essentially a DC resistivity method, but its device is a combined profile device. HX-GMD-01A integrates acquisition host, multi-channel electrode changeover switch, and high voltage conversion power supply to form a small light and portable system.

HDRM benefits from the advantages of the electrode layout to obtain large amounts of observation information efficiently. Through reasonable inversion analysis and interpretation algorithms, in areas difficult to explore by the conventional DC method. HDRM is usually applicable. However, when the buried depth of the pipeline is too large or the diameter of the pipeline is too small, the location accuracy will decrease. Moreover, the electrode layout in the early stage of this method is very

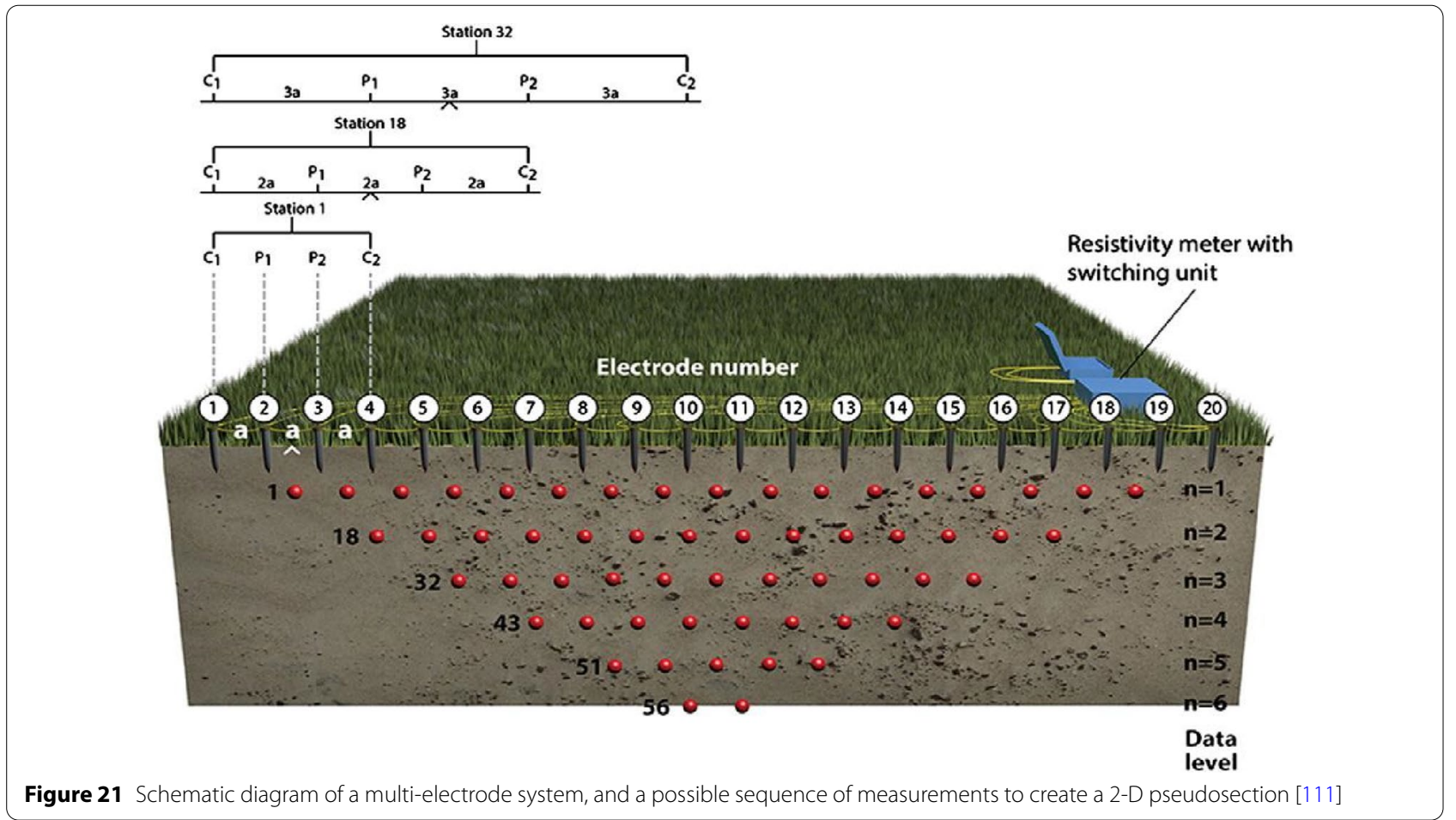


complicated. In the process of detecting buried nonmetallic pipelines, it is necessary to select appropriate electrode arrangement devices (Wenner, dipole, differential, Schlumberger device) according to different site characteristics to achieve the best location accuracy [111, 112]. Concerning the above problems, the research on the high-density resistivity method mainly includes the following two aspects:

(1) The ability of anti-jamming has been enhanced. The resolution and precision of location space have been improved. Although the high-density electrical method has higher resolution and higher efficiency than the conventional DC method, its location theoretical basis has not changed, and it is still a volume location method. To obtain a better location effect, it is necessary to select appropriate electrode spacing and array length. In modern cities, using the HDRM to detect buried non-metallic pipelines will inevitably encounter the influence of buried stray current [113], poor surfacing conditions, and sudden change of polarization potential difference. It is very important to remove interference and improve location accuracy.

(2) Related inversion analysis and interpretation algorithms have been researched. Wu et al. [119] found that when there is no water in the space, it is in a high resistance state. Otherwise, it is in a low resistance state. Phengnaone et al. [120] found that the increment of porosity and water saturation reduces the resistivity.

\subsection{Inertial Gyroscope}

The inertial gyroscope can accurately measure the three-dimensional coordinates of the pipeline [121, 122] depending on the movement of the heading and attitude sensors in the pipeline, as shown in Figure 22. When using the gyroscope, the measurement data will be transmitted to the whole location system after ensuring the direction of the rotation axis. Typical gyroscopes include Optical Gyroscope, Micro-Electromechanical System (MEMS) Gyroscope, Hemispherical Resonance Gyroscope (HRG), and Atomic Gyroscope, etc. [123, 124].

The inertial gyroscope is mainly used for the location of buried trenchless non-metallic pipelines with two ends open. It can record 3D information of pipelines permanently and accurately. At present, there are large-scale inertial navigation instruments with high tactical accuracy and small-size (less than 8-inch diameter) MEMS inertial measurement unit based on the Kalman filter [123]. The experimental test is shown in Figure 23.

The inertial intelligent Pipeline Inspection Gauge (PIG) [125] surveying technology was originally developed by Hanna [126] in the 1990s. During that period, the tactical-grade Fiber Optic Gyroscope (FOG) comprised of a

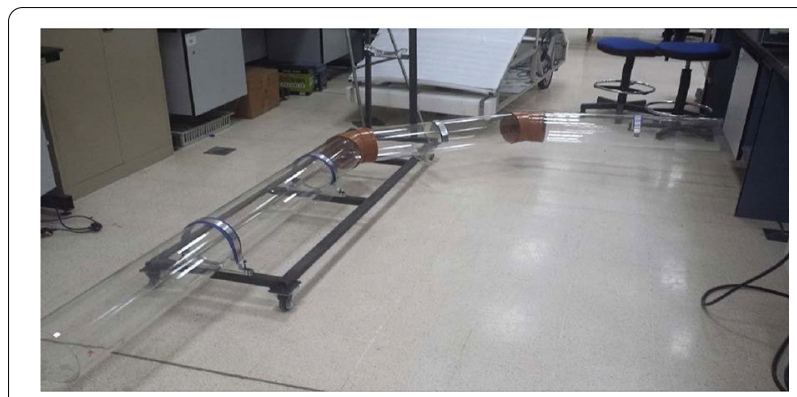

Figure 23 Used pipe in the experimental work [125]

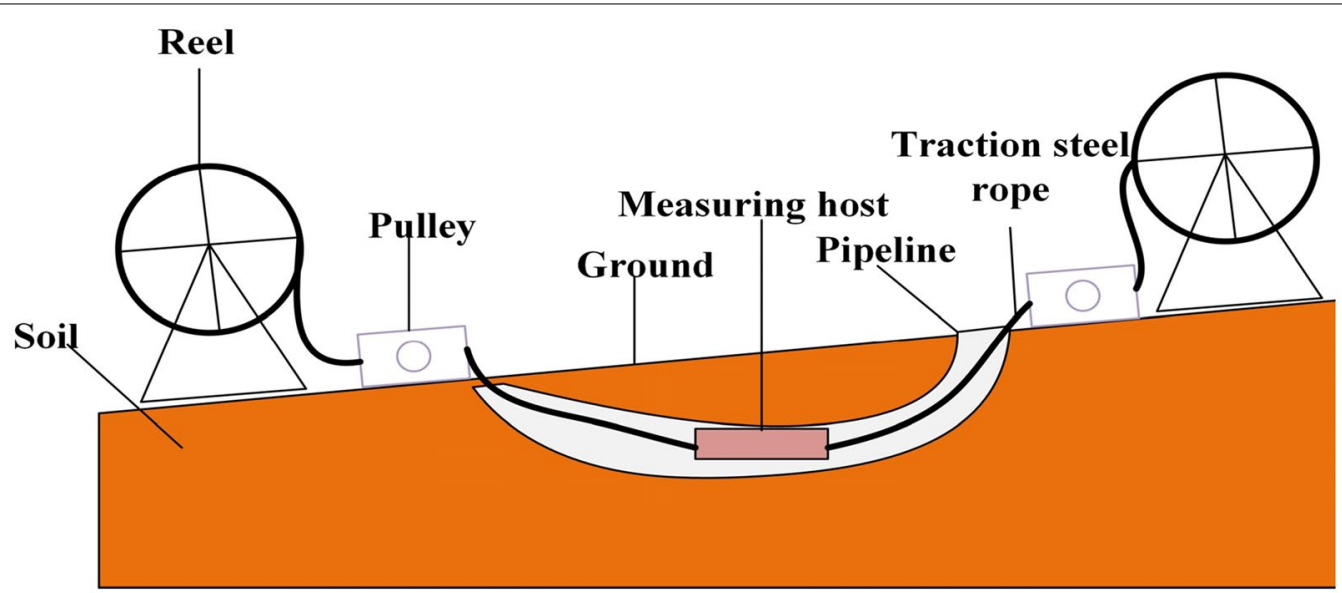

Figure 22 Layout of an Inertial gyroscope 
Strapdown Inertial Navigation System (SINS) is the best choice for the location of the PIG [127, 128]. However, these gyroscopes do not have high accuracy and fail to measure the pipeline with a small diameter. With the development of MEMS sensors these days, MEMS IMU can be used for the measurement of non-metallic pipelines with a small diameter [129-133].

The inertial gyroscope uses gyroscope location, inertial navigation, and computer three-dimensional simulation technologies. It can display the depth and direction of the buried pipeline on the computer in real-time, which is very intuitive and convenient. However, when using this method, the preparatory work is complex. It needs to stop the internal medium such as water and gas to open the two ends of the pipeline. To improve the measurement accuracy and reduce the complexity of implementation of the inertial gyroscope [121, 134], the following aspects of improvement and research are underway.

(1) Pipes with smaller diameters have been measured with an integrated inertial gyroscope measurement unit. The precision and real-time performance of MEMS inertial devices designed by Chen et al. [122] have reached the accuracy requirements of engineering.

(2) The algorithm of inertial gyroscope location measurement has been improved to enhance measurement accuracy. Shen et al. [121] proposed to use a linear trend modified by a self-organizing algorithm as a predictive model to improve the efficiency of the correction algorithm. The Riss algorithm proposed by AlMasri et al. [134] has better accuracy and smoother trajectory estimation than the full inertial navigation algorithm. A lower-order model and lower computational complexity are used in the reduced inertial sensor system (RISS) algorithm.

\subsection{Electrical Capacitance Tomography}

Electrical capacitance tomography (ECT) [135] is based on the different permittivity of each phase of the measured substances. When the distribution of the components of each phase changes, it will cause the equivalent dielectric constant to change, so that the capacitance value between the measuring electrode pairs will change. The corresponding image reconstruction algorithm [136] reconstructs the dielectric distribution map of the measured object field. ECT is divided into two types: circular [137] and planar. ECT image reconstruction involves two important calculation processes: the forward problem and the inverse problem. The location problem uses the known dielectric constant to solve the capacitance value between the electrode pairs.
The inverse problem uses the known capacitance data to estimate the dielectric constant distribution of the measured area. Locating buried non-metallic pipelines uses planar and inverse problem image reconstruction techniques. ECT is very sensitive to plastic and ceramic pipe materials. The film characteristics of the sensor make it suitable for many applications. Moreover, ECT provides greater penetration depth at a lower cost compared to GPR. A planar 16-electrode array to locate buried non-metallic pipes developed by BJ Huber et al. [138] is shown in Figure 24.

During the mid-1990s, Gas Technology Institute (GTI) has successfully located buried non-metallic pipelines by using an experimental capacitance scanning unit. Huber et al. [138] researched detecting and locating buried non-metallic pipes using electrical capacitance tomography (ECT) technology. Eight experimental technical documents were published on the information website [139]. Later scholars mainly studied image reconstruction algorithms, improving signal-to-noise ratio and optimizing sensors [140-142].

Currently, ECT is mainly used for non-destructive testing (NDT) [116] and pipeline location, etc. It has the advantages of low equipment cost, simple structure, and non-invasiveness.

However, there are still problems such as $[143,144]$ :

- The speed and accuracy of the image reconstruction algorithm cannot be improved in real-time.

- The dynamic range of the small capacitance measurement is large.

- The stray capacitance has influences on the capacitance measurement value.

- The ECT sensor design has an impact on the imaging effect.

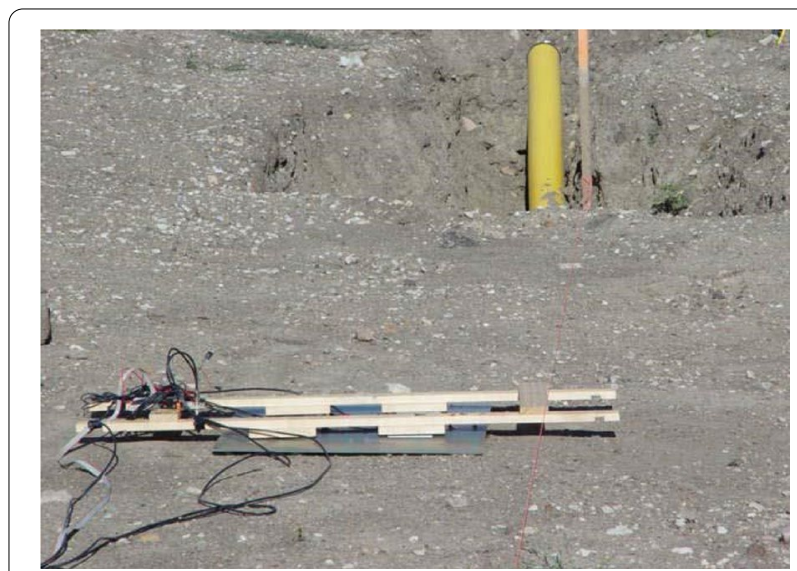

Figure 24 ECT sensor array plastic pipe in back surface exiting soil [139] 
To this end, researchers have conducted the following studies:

(1) The speed of image algorithm reconstruction has been improved. The L1 regularization-based second-order total variation (LSTV) algorithm proposed by G Guo et al. [140] ensures the sparsity of the reconstructed object, reduces the staircase effect brought by the first order total variation (FTV) regularization, and improves the estimation reliability. Cao et al. [145] proposed Calderon's method combined with a closed-loop control strategy for reconstructed images from noise-free data. This algorithm greatly improves the quality of reconstructed images.

(2) The signal-to-noise ratio (SNR) has been improved. Kryszyn et al. [142] found that using a switchable feedback capacitor in the integrator (first stage amplifier) increases the capacitor voltage gain. The signal-to-noise ratio and gain adjustment can be improved, and the measurement uncertainty will be improved in such a way that a single shot high voltage (SSHV) measurement value allows the reconstruction of high-quality images.

(3) The sensor design has been optimized to improve sensitivity. Tholin-Chittenden et al. [142] found that the symmetrical shape sensor has a better image reconstruction ability, and the study found that the measurement effect of the combined sensor is far better than that of the next best sensor.

Table 4 summarizes the strengths and weaknesses of other physics-based technologies of non-metallic pipeline detection and location. Infrared heat uses temperature differences to locate the pipeline, so this method can only be used to detect non-metallic pipelines such as heating, which has large limitations and low accuracy.
The electrode layout of the high-density resistivity method is complicated, cumbersome, and low in accuracy. Before the inertial gyroscope is used, it is necessary to stop the gas and excavate the buried non-metallic pipeline and place the measurement host in the pipeline with high accuracy. ECT uses planar array sensors to detect and locate buried non-metallic pipelines. It is suitable for any surface, but is easily affected by the magnetic field emitted by stray currents and has low accuracy.

Challenges for non-destructive evaluation (NDE) of buried pipelines include detection sensitivity of defects including compound defects, location and sizing of defects, efficiency of detection and location. Acoustic methods in locating underground pipelines have proven to be a potential supplement to the existing techniques for locating both metallic and plastic pipes. More and more multi-physical integrative NDT\&E including wide spectrum of electromagnetic methods are expected in the future to address the challenges.

\section{Emerging Relevant Technologies of Physics-Based Detection and Location}

\subsection{Geographic Information System}

Geographic Information System (GIS) [19, 146] is a data information system that describes, collects, stores, manages and analyzes the earth's surface, spatial, and geographical distribution. It is based on a geospatial database in the computer to combine with a variety of buried non-metallic pipeline detection and location technologies. To collect, manage, operate, analyze, simulate, and display spatially related data, it also uses the geographic model analysis method to provide a variety of spatial and dynamic geographic information in realtime for all kinds of research, comprehensive evaluation, management, and evaluation. A class of computer application systems are established by quantitative analysis and decision service [147]. GIS is a computer

Table 4 Summary of other physics-based detection and location technologies

\begin{tabular}{|c|c|c|c|c|c|}
\hline Methods & $\begin{array}{l}\text { Detection and Location } \\
\text { conditions }\end{array}$ & Accuracy & Interference & Strengths & Weaknesses \\
\hline Infrared thermography [18] & $\begin{array}{l}\text { Pipeline with high tempera- } \\
\text { ture }\end{array}$ & Low & Large & Easy to be operated & $\begin{array}{l}\text { Easily influenced by nearby } \\
\text { thermal sources }\end{array}$ \\
\hline HDRM [111] & Big resistivity difference & Low & Large & $\begin{array}{l}\text { The electrode arrangement } \\
\text { can be completed at one } \\
\text { time with high efficiency }\end{array}$ & $\begin{array}{l}\text { It is easy to be interfered } \\
\text { with by the outside; the data } \\
\text { processing and interpretation } \\
\text { are complex }\end{array}$ \\
\hline Inertial gyroscope [121] & $\begin{array}{l}\text { Open-ended non-metallic } \\
\text { pipeline }\end{array}$ & High & Small & $\begin{array}{l}\text { Plot and display the pipeline } \\
\text { depth and path real-timely }\end{array}$ & $\begin{array}{l}\text { The equipment is complex and } \\
\text { expensive, and the diameter } \\
\text { is limited by the size of the } \\
\text { gyroscope. }\end{array}$ \\
\hline ECT [135] & Any ground surface & Low & Large & $\begin{array}{l}\text { Low equipment cost, simple } \\
\text { structure }\end{array}$ & Vulnerable to stray current \\
\hline
\end{tabular}


system composed of software, hardware, and data describing geographic information and related ancillary information. The main difference between GIS and the map is that GIS can not only display a street but also show additional information, such as the name of the street, the time of pipe laying [148]. An example of the oil pipeline routes optimized by GIS model is shown in Figure 25.

The computer-based GIS began to develop in the late 1960s [148]. In the early stage of its development, it was difficult to detect and locate buried non-metallic pipelines without excavation [149]. With the development of GIS technology, the buried route, detection, and positioning, excavation, and maintenance of urban buried non-metallic pipelines [150, 151] have been improved.

GIS is mainly applied to the digitalization and intellectualization of pipelines in newly-built cities [152, 153], which is convenient and intuitive to display the trend and depth of buried non-metallic pipelines. For existing buried pipelines, this technology relies on several other buried pipeline location technologies and has been applied in Sanya city of Hainan Province and Transportadora de Gas del Peru (TGP).

GIS can be used to timely and conveniently search, and manage buried pipeline network data. So the damaged or aging pipes can be repaired or replaced in time. It secures water and gas uses. However, the data in time is necessitated in this method and it relies heavily on the algorithm $[19,146]$.

At present, mainly through the improvement of the pipe network management algorithm, the efficiency of pipe network management can be enhanced. The Computer Vision (CV) version of outdoor Augmented Reality GIS (ArGis) designed by Zhang et al. [147] has a good effect on virtual reality integration and interactive experience. A sewage pipeline risk ranking method based on the combination of spatial GIS and Analytic Hierarchy Process (AHP) - Data Envelopment Analysis

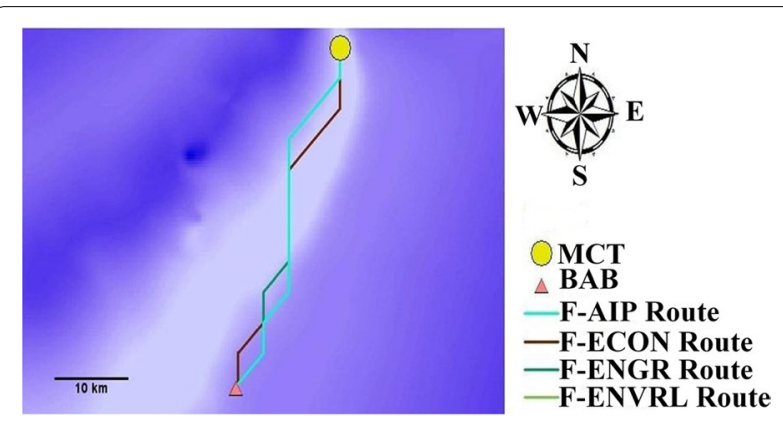

Figure 25 Overlay of all routes derived from fuzzified weights [19]
(DEA) proposed by Ghavami et al. [153] can well sort the repair of sewage pipe network and ensure the safety of pipe network operation.

\subsection{In-Pipe Robot}

As shown in Figure 26, the in-pipe robot $[126,154]$ applies small robots to pipelines with larger diameters and entrances and exits $[155,156]$. The in-pipe robot comes with its own power supply, camera, controller and GPS, and other positioning devices. The robot can walk and turn freely in the non-metallic pipeline through the controller and send the internal video signal and position information of the pipeline to the external host computer through the cable or wireless module.

In-pipe robots are used for internal pipeline damage detection and pipeline location. At present, In-pipe robots have mature products such as TV-150 wheeled robots developed by China information technology and German TV-150 wheeled robots.

In urban public utilities, pipeline robots play an extremely important role in the detection and positioning of buried non-metallic pipelines [157]. A pipeline robot is used in horizontal directional drilling (HDD) projects to detect and locate pipelines [158, 159]. The friction between the robot and the pipeline is enhanced by increasing the wheel [160]. However, the degree of freedom and the complexity of the control strategy also increases. Therefore, the magnetic wheel pipeline robot appears, but it is only applicable to metal pipelines [161, $162]$. With the development of pipeline robots, the detection and positioning of buried non-metallic pipelines are more accurate [163].

The in-pipe robot has a high location accuracy. It can detect the damage inside the pipeline and send the location signal to be received by the computer. However, this method is limited by the size of the robot as well as its turning and walking ability in the pipeline $[6,157]$. The improvements and researches mainly focused on the following aspects:

(1) The environmental adaptability of the robot locator has been improved. The traction experiment of the active screw in-pipe robot designed by $\mathrm{Tu}$ et al. [163] proved that the increase of the adhesion coefficient and the decrease of the driving wheel skid will increase the traction force, making the robot move faster. Kakogawa et al. [164] introduced a robot with a novel foldable Omni-Crawler module. The modules are connected through passive joints through links, which makes the overall movement possible, which facilitates the alignment of the robot in the bending direction. 

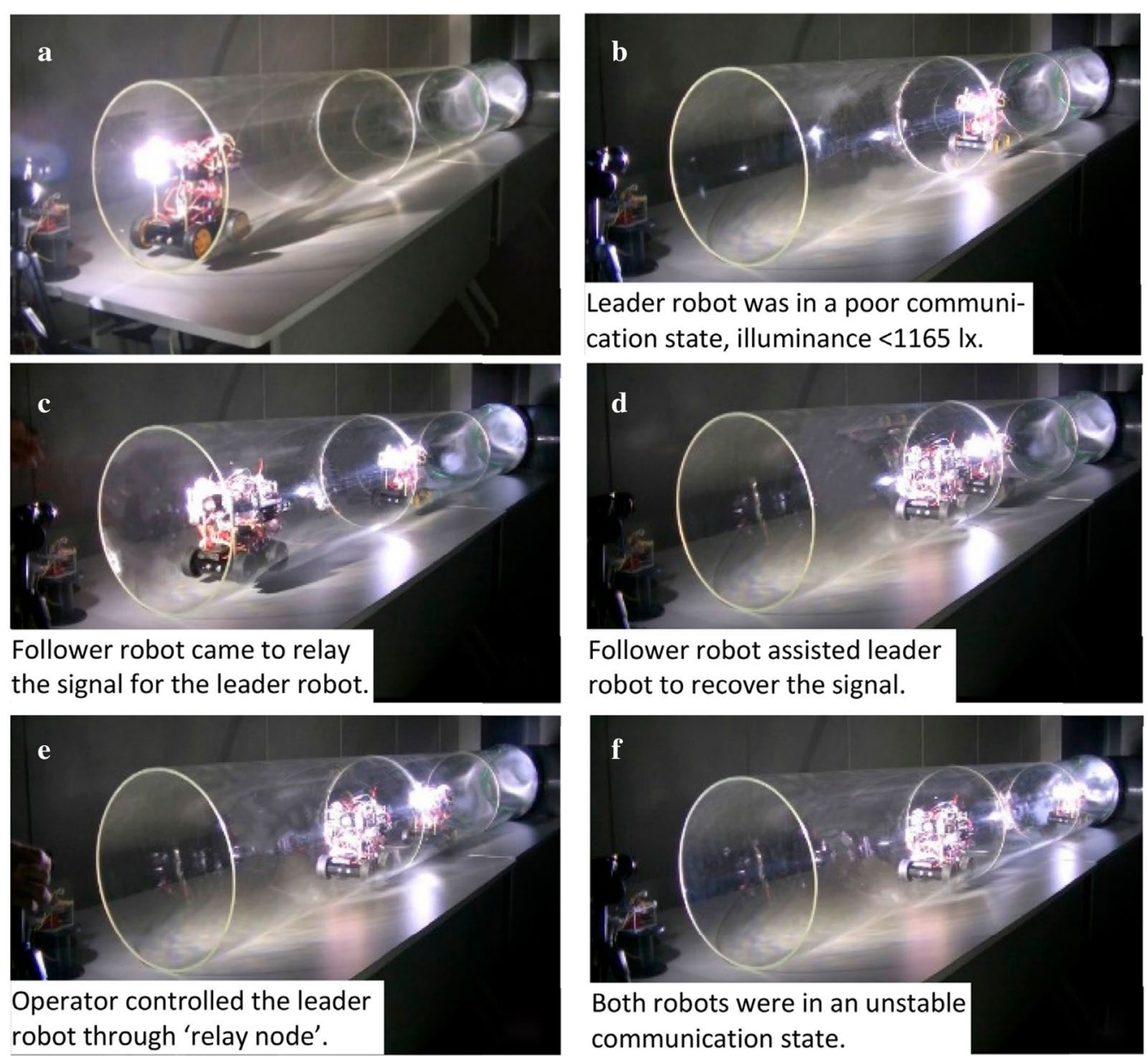

Figure 26 Coordinated movement of Robot Chain System (RCS) in transparent pipe [155]

(2) The robot location algorithm has been improved. The algorithm designed by Kennedy et al. [154] proposed a novel single-view representation of information from a multi-camera system using simple time filtering to achieve a high-precision location. Zhao [155] of Waseda University developed visible light relay communication to enhance communication capability.

\subsection{Other Emerging Technologies}

With the emergence of new technologies, many other emerging technologies have appeared for the detection and positioning of buried non-metallic pipelines.

(1) The static electromagnetic field method is designed for detecting and locating the abnormal changes of the earth [165]. It is based on the principle that the static electromagnetic field varies according to the heterogeneity of the soil. The detecting system is composed of a transmitter, grounding electrode, signal intensifier, receiving antenna, and other components. It can be used to judge the presence, location, direction, and diameter of buried non-metallic pipelines.

(2) The space satellite [166] is used to identify the signs (surge tank, identification column, well cover, etc.) of obvious buried non-metallic pipelines in streets and fields. An intelligent algorithm is proposed to infer the path of buried non-metallic pipelines.

(3) The ultrasonic guided wave generated by the pulse generator [167] moves radially along the two sides of the non-metallic pipeline. It can detect the depth and direction of the buried non-metallic pipeline at the same time. International researchers have spent a great number of efforts on developing the detection and location of buried non-metallic pipelines by ultrasonic method.

(4) Near-field microwave holographic imaging is augmented with beamspace transformation [168, 169]. 
It can be used to create the 3-D image of multiple concentric nonmetallic pipes by measuring the backscattered fields in the near-field region. In addition, the use of beamspace transformation allows for improving the images of the defects on the inner pipes, i.e., pipes are farther away from the measuring antennas.

Table 5 summarizes the strengths and weaknesses of the emerging detection and location technologies for non-metallic pipelines. GIS is a kind of geospatial information management system, which combines buried pipeline data from various detection methods and urban geographic information systems. It can easily and quickly determine the depth and direction of urban buried pipelines on the host computer interface. Only personnel is allowed to update the database in real-time with high accuracy. The in-pipe robot provides the internal conditions and the positioning information of the pipeline with high accuracy. Other emerging technologies are effective supplements to the detection and positioning of buried non-metallic pipelines with imperfect theory and low accuracy.

\section{Guide for Selection of Detection and Location Methods of Buried Non-Metallic Pipelines}

As mentioned in previous sections, there are various methods and mechanisms for the location and detection of buried non-metallic pipelines. However, the applicability of each method varies greatly according to the working conditions of the pipeline, the characteristics of the pipeline, and the medium location methods inside and outside the pipelines. For example, the location and depth of buried non-metallic pipelines can be detected on the surface and in the pipe by using methods such as the electromagnetic tracer method, Infrared thermography method, and Inertial gyroscope. The Infrared thermography method can only be applied to a buried heating pipeline network location. The elastic wave method can be applied to all types of buried nonmetallic pipelines, including oil and natural gas, and water. HDRM and ECT are both non-destructive tomography systems for visual detection and positioning of buried non-metallic pipelines. However, some methods can only detect non-metallic hanging pipelines with specific depth. To provide criteria for selecting methods suitable for specific situations, Table 6 lists the main available location technologies and selection criteria for buried non-metallic pipelines. The location methods should be selected according to the actual location conditions.

\section{Conclusions and Future Perspectives}

The detection of the directions and positions of the buried pipelines is especially important. Currently, nondestructive testing, especially the global physical location technology is getting mature. Higher requirements for non-metallic pipeline locations have been brought about, for example, the requirements for different soils, the different depths, and electromagnetic radiations. Therefore, current buried non-metallic pipeline detection and location technologies need to be upgraded and transformed with new technology and innovation. The research in the future is aiming at a new location method of non-metallic pipelines and achieving this for a combination of various location methods in line with other pipeline inspection and monitoring [170]. The research and development trends for non-metallic pipeline detection and location can be foreseen according to the previous analysis, as summarized in the following.

(1) Multi-sensor and multi-feature fusion for location and leakage detection of a non-metallic pipeline should be carried out along with new sensor technologies. At present, the location detection technology of non-metallic buried pipelines is based on different physical principles has certain advantages and disadvantages. Combining these methods, the situation of the buried non-metallic pipeline can be

Table 5 Summary of emerging detection and location technologies

\begin{tabular}{|c|c|c|c|c|c|}
\hline Methods & $\begin{array}{l}\text { Detection and Location } \\
\text { conditions }\end{array}$ & Accuracy & Interference & Strengths & Weaknesses \\
\hline GIS [19] & Streets with GIS & High & Small & $\begin{array}{l}\text { Easy operation, high accuracy, } \\
\text { 3D display available }\end{array}$ & $\begin{array}{l}\text { Updating topographical data } \\
\text { real-time; high expense for } \\
\text { maintenance }\end{array}$ \\
\hline In-pipe robot [164] & $\begin{array}{l}\text { Open-ended non-metallic } \\
\text { pipeline }\end{array}$ & High & Small & $\begin{array}{l}\text { Locate accurately and easily } \\
\text { access all conditions inside the } \\
\text { pipeline }\end{array}$ & $\begin{array}{l}\text { Limited by the size of the robot } \\
\text { and its ability to turn and com- } \\
\text { municate. }\end{array}$ \\
\hline $\begin{array}{l}\text { Other emerging } \\
\text { technologies [165- } \\
167]\end{array}$ & $\begin{array}{l}\text { Various environments and } \\
\text { surface }\end{array}$ & Low & Large & $\begin{array}{l}\text { A beneficial supplement to the } \\
\text { existing non-metallic pipeline } \\
\text { detection methods }\end{array}$ & $\begin{array}{l}\text { The theory of emerging tech- } \\
\text { nologies is not perfect, and the } \\
\text { equipment is unstable }\end{array}$ \\
\hline
\end{tabular}


Table 6 Selection guide for detection and location methods of buried non-metallic pipelines

\begin{tabular}{|c|c|c|c|}
\hline Methods & Scope of application & Methods & Scope of application \\
\hline \multicolumn{4}{|c|}{ Electromagnetic tracer line method } \\
\hline Direct connection & $\begin{array}{l}\text { Tracer lines are laid on the surface of } \\
\text { non-metallic pipes }\end{array}$ & Inertial gyroscope & $\begin{array}{l}\text { The diameter of the inertial naviga- } \\
\text { tion unit is smaller than the diameter } \\
\text { of the non-metallic pipe }\end{array}$ \\
\hline \multirow[t]{2}{*}{ Induction } & & $\mathrm{ECT}$ & Any surface \\
\hline & & Pipe excitation method & $\begin{array}{l}\text { Non-metallic pipes are exposed on } \\
\text { the surface }\end{array}$ \\
\hline Tracer probe method & $\begin{array}{l}\text { Probe diameter is smaller than the } \\
\text { diameter of non-metallic pipe }\end{array}$ & Elastic wave method & Any surface \\
\hline GPR & $\begin{array}{l}\text { Any surface, the image has multiple } \\
\text { solutions }\end{array}$ & Point vibration measurement & Soft surface \\
\hline RFID & $\begin{array}{l}\text { Electronic tags are buried near non- } \\
\text { metallic pipes }\end{array}$ & GIS & $\begin{array}{l}\text { Towns where buried non-metallic } \\
\text { pipelines have been digitized }\end{array}$ \\
\hline Infrared thermography & $\begin{array}{l}\text { Non-metallic pipes containing high- } \\
\text { temperature fluid }\end{array}$ & In-pipe Robot & $\begin{array}{l}\text { Robot diameter is smaller than the } \\
\text { non-metallic pipe diameter }\end{array}$ \\
\hline HDRM & Any surface & Other emerging technologies & Developing stage \\
\hline
\end{tabular}

comprehensively analyzed. In addition, the problem of pipeline location and tracking under complex background can be solved, especially the problem of the coexistence of metal and non-metallic pipelines.

(2) Emerging technology of data analysis, signal processing, and image reconstruction is required. Artificial intelligence technology and decision-making should be combined. At the same time, the numerical models of time, space, soil, and field require to be established. It effectively simulates the buried depth and path of the buried non-metallic pipeline, in order to realize the multi-dimensional visualization of non-metallic pipeline location.

(3) Theoretical analysis and numerical simulation combined with new materials, new theories, and the new location method of a non-metallic pipeline need to be conducted. Combined with geographical exploration and drilling technology, the system can be formed for real-time detection of the non-metallic pipeline. The accuracy and limitation of the location depth will also increase. The non-destructive location and evaluation ability are quite necessary to identify the damages and health conditions. For instance, stress, integrity management, fault early warning, and safe operation service require to be contained.

(4) Establishment and improvement of GIS for buried pipeline management is a new trend. Automatic measurement, data storage, intuitive results display, and interpretation automation technology require to be reasonably used. The integrated operation mode is used. The achievements of buried pipeline location are applied to the urban buried pipe network management GIS. The corresponding terrain database, pipeline database, comprehensive database, etc., are well established.

(5) The development trends of buried location equipment include low-cost, miniaturization, modularization, easy operation, low-power consumption, multi-functional portable network, embedded monitoring, etc. The design of a small structure hardware system can enhance the adaptability and convenience of the system in a complex environment. The module function is refined to continuously improve the reliability and maintainability of the system. The equipment cost and power consumption are reduced, while the operational performance is improved. This makes it conducive to the promotion, application, digital intelligent location, monitoring, and management of the equipment.

\section{Authors' Contributions}

$L G, G T, X X, G W, Z H$, JX, and MR were all in charge of the whole trial; CZ wrote the manuscript; GT and JA assisted with the revision. All authors have read and approved the final manuscript.

\section{Authors' Information}

Liang Ge is currently an associate professor at School of Mechanical and Electrical Engineering, Southwest Petroleum University, China.

Changpeng Zhang is currently a postgraduate at School of Mechanical and Electrical Engineering, Southwest Petroleum University, China.

Guiyun Tian is currently a professor at School of Engineering, Newcastle University, UK.

Xiaoting Xiao is currently a lecturer at School of Electrical Information, Southwest Petroleum University, China. 
Guohui Wei is currently a lecturer at School of Science, Southwest Petroleum University, China.

Ze Hu is currently a professor at School of Electrical Information, Southwest Petroleum University, China.

Junaid Ahmed is currently an assistant professor at Electrical Department of Sukkur IBA University, Sukkur, Sindh, Pakistan.

Ju Xiang is a MSc candidate at the School of Engineering, Newcastle University. Mark Robinson is a professor at School of Engineering, Newcastle University, UK.

\section{Funding}

Supported by Downhole Intelligent Measurement and Control Science and Technology Innovation Team of Southwest Petroleum University (Grant No. 2018CXTD04), National Natural Science Foundation of China (Grant Nos. 61701085, 51974273), Chengdu Municipal international science and technology cooperation project of China (Grant Nos. 2020-GH02-00016-HZ) and 2020 National Mountain Highway Engineering Technology Research Center Open Fund Project (Grant No. GSGZJ-2020-01).

\section{Competing Interests}

The authors declare no competing financial interests.

\section{Author Details}

'School of Mechanical and Electrical Engineering, Southwest Petroleum University, Chengdu 610500, China. ${ }^{2}$ School of Engineering, Newcastle University, Newcastle NE1 7RU, UK. ${ }^{3}$ School of Electrical Information, Southwest Petroleum University, Chengdu 610500, China. ${ }^{4}$ Electrical Department, Sukkur IBA University, Sukkur 65200, Pakistan. ${ }^{5}$ School of Science, Southwest Petroleum University, Chengdu 610500, China.

Received: 3 November 2020 Revised: 31 March 2021 Accepted: 2 September 2021

Published online: 18 October 2021

\section{References}

[1] U Baroudi, A A Al-Roubaiey, A Devendiran. Pipeline leak detection systems and data fusion: A survey. IEEE Access, 2019, 7: 97426-97439.

[2] Y Zhang, S Huang, W Zhao, et al. Electromagnetic ultrasonic guided wave long-term monitoring and data difference adaptive extraction method for buried oil-gas pipelines. International Journal of Applied Electromagnetics and Mechanics, 2017, 54(3): 329-339.

[3] TWang, J Cao, L Pei. A novel underground pipeline surveillance system based on hybrid acoustic features. IEEE Sensors Journal, 2020, 21 (2): 1040-1050.

[4] TS T Amran, M S M Amin, M R Ahmad, et al. A study on detection water leakage of underground metal and PVC pipes using ground penetrating radar. International Nuclear Science Technology and Engineering Conference, Skudai, Malaysia, November 23-25, 2018, 555(1): 012012

[5] R Khademi-Zahedi. Application of the finite element method for evaluating the stress distribution in buried damaged polyethylene gas pipes. Underground Space, 2019, 4(1): 59-71.

[6] H Lu, J Matthews, T Iseley. How does trenchless technology make pipeline construction greener? A comprehensive carbon footprint and energy consumption analysis. Journal of Cleaner Production, 2020: 121215.

[7] H R Vanaei, A Eslami, A Egbewande. A review on pipeline corrosion, in-line inspection (ILI), and corrosion growth rate models. International Journal of Pressure Vessels and Piping, 2017, 149: 43-54.

[8] M Karami. Review of corrosion role in gas pipeline and some methods for preventing it. Journal of Pressure Vessel Technology, 2012, 134(5): 1-5.

[9] B Hu, R Yu, J Liu. Experimental study on the corrosion testing of a buried metal pipeline by transient electromagnetic method. Anti-Corrosion Methods and Materials, 2016, 63(4): 262-268.

[10] Q Feng, R Li, B Nie, et al. Literature review: Theory and application of in-line inspection technologies for oil and gas pipeline girth weld defection. Sensors, 2017, 17(1): 50.

[11] Z Long, W Wei, A Turlapaty, et al. Fusion of radiation and electromagnetic induction data for buried radioactive target detection and characterization. IEEE Transactions on Nuclear Science, 2013, 60(2): 1126-1133.

[12] B A Sundaram, K Kesavan, S Parivallal. Recent advances in health monitoring and assessment of in-service oil and gas buried pipelines. Journal of The Institution of Engineers (India): Series A, 2018, 99(4): 729-740.

[13] M A Volosova, A A Okunkova, SV Fedorov, et al. Electrical discharge machining non-conductive ceramics: combination of materials. Technologies, 2020, 8(2): 32

[14] S Datta, S Sarkar. A review on different pipeline fault detection methods. Journal of Loss Prevention in the Process Industries, 2016, 41: 97-106.

[15] I Galili, D Kaplan, Y Lehavi. Teaching Faraday's law of electromagnetic induction in an introductory physics course. American Journal of Physics, 2006, 74(4): 337-343.

[16] V Chandrasekar, H Chen, H Tan, et al. Rainfall estimation from ground radar and TRMM Precipitation Radar using hybrid deep neural networks. Geophysical Research Letters, 2019, 46(17-18): 10669-10678.

[17] Y Zhang, J Shi. Design of polyethylene ventilation pipeline leakage monitoring system based on fusion of multiple signal sources. Earth and Environmental Science, 2020, 440(2): 022107.

[18] L Shao, Y M Zhang, J Li, et al. Research on high temperature region segmentation of infrared pipeline image based on improved two-dimensional-Otsu. Spectroscopy and Spectral Analysis, 2019, 39(5): 1637-1642.

[19] A L Balogun, A N Matori, A I Hamid-Mosaku, et al. Fuzzy MCDM-based GIS model for subsea oil pipeline route optimization: An integrated approach. Marine Georesources \& Geotechnology, 2017, 35(7): 961-969.

[20] Radiodetection Ltd. ABC\& XYZ of locating buried pipes and cables for the beginner and the specialist. UK: Radiodetection Ltd, 2008.

[21] C H Xu, H B Zhu, N F Zhu. Tracer wire in non-metallic pipeline detection. Urban Geotechnical Investigation \& Surveying, 2011, (01): 159-163. (in Chinese)

[22] F L H Cavalcante, C Schamper, C Noel, et al. Geometrical characterization of urban fill by integrating the multi-receiver electromagnetic induction method and electrical resistivity tomography: A case study in Poitiers, France. European Journal of Soil Science, 2019, 70(5): 1012-1024.

[23] Y Chen, B Z Wang. Four-domain dual-combination operation invariance and time reversal symmetry of electromagnetic fields. Optics Express, 2013, 21(21): 24702-24710.

[24] G E Qady, M Metwaly, A Khozaym. Tracing buried pipelines using multi frequency electromagnetic. NRIAG Journal of Astronomy and Geophysics, 2014, 3(1): 101-107.

[25] Z F Cao. Tracer line method of gas pe pipelines and its detection technique. Site Investigation Science and Technology, 2010(04): 61-64. (in Chinese)

[26] D Ambruš, D Vasić, V Bilas. Robust estimation of metal target shape using time-domain electromagnetic induction data. IEEE Transactions on Instrumentation and Measurement, 2016, 65(4): 795-807.

[27] L Ma, HY Wei, M Soleimani. Pipelines inspection using magnetic induction tomography based on a narrowband pass filtering method. Progress in Electromagnetics Research, 2012, 23: 65-78.

[28] P Chen. CJJ 61-2017 Technical specification for urban underground pipeline detection. Beijing: Beijing Surveying and Mapping Technology Design and Research Institute, 2017. (in Chinese)

[29] F A Karaa, A Katz, E Niver. Decision analysis of preferred methods for locating underground conduits. Journal of Pipeline Systems Engineering and Practice, 2014, 5(2): 04013017.

[30] S Rudolph, B P Marchant, L Weihermüller, et al. Assessment of the position accuracy of a single-frequency GPS receiver designed for electromagnetic induction surveys. Precision Agriculture, 2019, 20(1): 19-39.

[31] HWang, Q Zhu, Z Ding, et al. A fully-packaged ship-shaped hybrid nanogenerator for blue energy harvesting toward seawater self-desalination and self-powered positioning. Nano Energy, 2019, 57: 616-624.

[32] D Mishra, K K Agrawal, A Abbas, et al. PIG [Pipe Inspection Gauge]: An artificial dustman for cross country pipelines. Procedia Computer Science, 2019, 152: 333-340.

[33] B Uslu, Y J Jung, S K Sinha. Underground utility locating technologies for condition assessment and renewal engineering of water pipeline infrastructure systems. Journal of Pipeline Systems Engineering and Practice, 2016, 7(4): 04016011. 
[34] L L Chen. Application of tracer method in underground pipeline exploration under complicated conditions. Science and Technology of West China, 2011, 10(25): 22-23. (in Chinese)

[35] J Dexmer, C M Leroy, L Binet, et al. Vanadium Oxide- PANI nanocomposite-based macroscopic fibers: $1 \mathrm{~d}$ alcohol sensors bearing enhanced toughness. Chemistry of Materials, 2008, 20(17): 5541-5549.

[36] S Roshani, M Hayati, S Setayeshi, et al. A miniaturized harmonic suppressed power amplifier integrated with lowpass filter for long term evolution application. Analog Integrated Circuits and Signal Processing, 2016, 89(1): 197-204.

[37] S Miura, T Nakagami, V Parque, et al. Bi-rigid guide wire enables endoscope insertion into winding small gas pipelines. Mechanical Engineering Journal, 2020, 7(4):20-00088.

[38] H M Kim, H R Yoo, G S Park. A new design of MFL sensors for self-driving NDT robot to avoid getting stuck in curved underground pipelines. IEEE Transactions on Magnetics, 2018, 54(11): 1-5.

[39] M Starý, F Novotný, M Horák, et al. Sampling robot for primary circuit pipelines of decommissioned nuclear facilities. Automation in Construction, 2020, 119: 103303.

[40] M P Matos, M von Sperling, A T Matos, et al. Clogging in constructed wetlands: Indirect estimation of medium porosity by analysis of ground-penetrating radar images. Science of the Total Environment, 2019, 676: 333-342.

[41] L Langhammer, L Rabenstein, A Bauder, et al. Ground-penetrating radar antenna orientation effects on temperate mountain glaciers. Geophysics, 2017, 82(3): H15-H24.

[42] J Kavi, U B Halabe, HV S GangaRao. Detection of buried FRP composite pipes using ground penetrating radar. Journal of Multidisciplinary Engineering Science and Technology, 2020, 6(7): 10479-10484.

[43] Q Hoarau, G Ginolhac, A M Atto, et al. Robust adaptive detection of buried pipes using GPR. Signal Processing, 2017, 132: 293-305.

[44] R Salvi, A Ramdasi, Y A Kolekar, et al. Use of ground-penetrating radar (GPR) as an effective tool in assessing pavements-A review. Geotechnics for Transportation Infrastructure, 2019: 85-95.

[45] X Zeng, G A McMechan. GPR characterization of buried tanks and pipes. Geophysics, 1997, 62(3): 797-806.

[46] A Shaari, R S Ahmad, TH Chew. Effects of antenna-target polarization and target-medium dielectric contrast on GPR signal from non-metal pipes using FDTD simulation. NDT \& E International, 2010, 43(5): 403-408.

[47] E Yigit, S Demirci, C Ozdemir, et al. A synthetic aperture radar-based focusing algorithm for B-scan ground penetrating radar imagery. Microwave and Optical Technology Letters, 2007, 49(10): 2534-2540.

[48] J Zhou, Y Wang, Q Zhang. Research of geologic radar in anti-sliding piles inspection. 2nd International Conference on Remote Sensing, Environment and Transportation Engineering, Nanjing, China, June 1-3, 2012: $1-4$

[49] X Dou, L J Wei, D R Magee, et al. 3D buried utility location using a marching-cross-section algorithm for multi-sensor data fusion. Sensors, 2016, 16(11): 1-24.

[50] C Srinivas, T Srinivasarao. Buried pipes detection through ground penetrating radar images. International Journal of Mechanical Engineering and Technology, 2017, 8(12): 61-68.

[51] H Harkat, A E Ruano, M G Ruano, et al. GPR target detection using a neural network classifier designed by a multi-objective genetic algorithm. Applied Soft Computing, 2019, 79: 310-325.

[52] J Prinsloo, R Malekian. Accurate vehicle location system using RFID, an internet of things approach. Sensors, 2016, 16(6): 825.

[53] J Zhang, GYTian, A M J Marindra, et al. A review of passive RFID tag antenna-based sensors and systems for structural health monitoring applications. Sensors, 2017, 17(2): 265.

[54] V Khokhlova, E Delevoye. The localization of buried objects in the soil using an RFID tag: protocol description and parameter estimation with the model of the oscillating magnetic field in media. IEEE Transactions on Magnetics, 2019, 55(5): 1-16.

[55] R Vyas, B Tye. A sequential RFID system for robust communication with underground carbon steel pipes in oil and gas applications. Electronics, 2019, 8(12): 1374.

[56] C Z Wu, XY Wang, M C Chen, et al. Differential received signal strength based RFID positioning for construction equipment tracking. Advanced Engineering Informatics, 2019, 42: 100960.
[57] C Sun. Application of RFID technology for logistics on internet of things. AASRI Procedia, 2012, 1: 106-111.

[58] M H Zarifi, S Deif, M Daneshmand. Wireless passive RFID sensor for pipeline integrity monitoring. Sensors and Actuators A: Physical, 2017, 261: 24-29.

[59] Y C Lin, W F Cheung, F C Siao. Developing mobile 2D barcode/RFIDbased maintenance management system. Automation in Construction, 2014, 37: 110-121.

[60] N Outteridge. Internet of things security: A review on the RFID contactless security protocols. PeerJ Preprints, 2018. https://peerj.com/prepr ints/26504/

[61] A Narayanan, S Singh, M Somasekharan. Implementing RFID in library: methodologies, advantages and disadvantages. Recent Advances in Information Technology, 2005, 271.

[62] K Chen, Y Chen, W J Hsueh, et al. The study of an adaptively active control on the acoustic propagation in a pipe. Applied Acoustics, 1998, 55(1): 53-66.

[63] F C Lobato de Almeida. Improved acoustic methods for leak detection in buried plastic water distribution pipes. Southampton: University of Southampton, 2013.

[64] S Sazesh, S Shams. Vibration analysis of cantilever pipe conveying fluid under distributed random excitation. Journal of Fluids and Structures, 2019, 87: 84-101.

[65] Baghalian A, Tahakori S, Fekrmandi H, et al. Implementation of the surface response to excitation method for pipes. Mechanics of Composite and Multi-functional Materials, 2017, 7: 261-266.

[66] STashakori, A Baghalian, M Unal, et al. Contact and non-contact approaches in load monitoring applications using surface response to excitation method. Measurement, 2016, 89: 197-203.

[67] J M Muggleton, M J Brennan, Y Gao. Determining the location of buried plastic water pipes from measurements of ground surface vibration. Journal of Applied Geophysics, 2011, 75(1): 54-61.

[68] A Martini, A Rivola, M Troncossi. Autocorrelation analysis of vibro-acoustic signals measured in a test field for water leak detection. Applied Sciences, 2018, 8(12): 2450.

[69] F C L Almeida, M J Brennan, P F Joseph, et al. Towards an in-situ measurement of wave velocity in buried plastic water distribution pipes for the purposes of leak location. Journal of Sound and Vibration, 2015, 359: 40-55.

[70] HV Fuchs, R Riehle. Ten years of experience with leak detection by acoustic signal analysis. Appl. Acoust., 1991, 33(1): 1-19

[71] A Katz, F Karaa, E Niver. Innovative and effective techniques for locating underground conduits. New Jersey: University Transportation Research Center, 2011.

[72] J M Muggleton, M Brennan. The use of acoustic methods to detect and locate underground piping systems. The Ninth International Conference on Recent Advances in Structural Dynamics (RASD2006), Southampton, UK, November 17-19, 2006: 1-4.

[73] J M Muggleton, M J Brennan. The design and instrumentation of an experimental rig to investigate acoustic methods for the detection and location of underground piping systems. Applied Acoustics, 2008, 69(11): 1101-1107.

[74] CXu. Research on buried non-metallic pipe detection technology based on sound wave propagation characteristics. Huainan: Anhui University of Science and Technology, 2019. (in Chinese)

[75] J M Muggleton, J Yan. Wavenumber prediction and measurement of axisymmetric waves in buried fluid-filled pipes: Inclusion of shear coupling at a lubricated pipe/soil interface. Journal of Sound and Vibration, 2013, 332(5): 1216-1230.

[76] Y Gao, J M Muggleton, Y Liu, et al. An analytical model of ground surface vibration due to axisymmetric wave motion in buried fluid-filled pipes. Journal of Sound and Vibration, 2017, 395: 142-159.

[77] Y Gao, J M Muggleton, E Rustighi, et al. Ground surface vibration due to axisymmetric wave motion in buried fluid-filled pipes, International Congress on Sound \& Vibration, Florence, Italy, July 12-16, 2015: 1-8.

[78] Y Gao, Y Liu, J M Muggleton. Axisymmetric fluid-dominated wave in fluid-filled plastic pipes: Loading effects of surrounding elastic medium. Applied Acoustics, 2017, 116: 43-49.

[79] Y Gao, F S Sui, J M Muggleton, et al. Simplified dispersion relationships for fluid-dominated axisymmetric wave motion in buried fluid-filled pipes. Journal of Sound and Vibration, 2016, 375: 386-402. 
[80] A Rahaman, C H Park, B Kim. Design and characterization of a MEMS piezoelectric acoustic sensor with the enhanced signal-to-noise ratio. Sensors and Actuators A: Physical, 2020: 112087.

[81] P Arumbu, S Srinivasalu. Sustainable model for high signal to noise ratio to measure underwater acoustic signal using Acoustic Doppler Velocimeter. Computers \& Electrical Engineering, 2018, 68: 262-270.

[82] J M Muggleton. Remote tree root mapping using a tree trunk vibration. The ISMA2014 including USD, Leuven, Belgium, September 15-17, 2014: 3807-3818.

[83] M K Kalkowski, J M Muggleton, E Rustighi. Tree root detection from ground surface vibration measurements. The MATEC Web of Conferences, Ho Chi Minh City, Vietnam, March 2-5, 2018: 1-5.

[84] B Papandreou, E Rustighi, M J Brennan. A study into the feasibility of using acoustic techniques to locate buried objects. Southampton: Institute of Sound and Vibration Research, University of Southampton, 2008.

[85] B Papandreou, M J Brennan, E Rustighi. On the detection of objects buried at a shallow depth using seismic wave reflections. The Journal of the Acoustical Society of America, 2011, 129(3): 1366-1374.

[86] J M Muggleton, B Papandreou. A shear wave ground surface vibration technique for the detection of buried pipes. Journal of Applied Geophysics, 2014, 106: 164-172.

[87] V Ganji, N Gucunski, A Maher. Detection of underground obstacles by SASW method-Numerical aspects. Journal of Geotechnical and Geoenvironmental Engineering, 1997, 123(3): 212-219.

[88] H Dong, J Lai, P Li. Inverse obstacle scattering for elastic waves with Phased or phaseless far-field data. SIAM Journal on Imaging Sciences, 2019, 12(2): 809-838

[89] X Tian, W Zhao, Y Du, et al. Detection of mortar defects in ballastless tracks of high-speed railway using transient elastic wave method. Journal of Civil Structural Health Monitoring, 2018, 8(1): 151-160.

[90] P Shokouhi, N Gucunski, A Maher. Application of wavelets in detection of cavities under pavements by surface waves. Transportation Research Record, 2003, 1860(1): 57-65.

[91] J Lin, C Zhang, L L Sun, et al. Simulation of seismic wave scattering by embedded cavities in an elastic half-plane using the novel singular boundary method. Advances in Applied Mathematics and Mechanics, 2018, 10(2): 322-342.

[92] C B Xiong, Z Li, X Sun, et al. Integrated acoustic survey method for inspecting the unburied status of submarine pipeline. Journal of Hohai University (Natural Sciences), 2017, 45(5): 425-431. (in Chinese)

[93] A Van Pamel, G Sha, S I Rokhlin, et al. Finite-element modelling of elastic wave propagation and scattering within heterogeneous media. Proceedings of the Royal Society A: Mathematical, Physical and Engineering Sciences, 2017, 473(2197): 20160738.

[94] L Karl, W Haegeman, G Degrande. Determination of the material damping ratio and the shear wave velocity with the Seismic Cone Penetration Test. Soil Dynamics and Earthquake Engineering, 2006, 26(12): 1111-1126.

[95] T Sugimoto, M Okujima. Underground Imaging Using Shear Waves: Stacking Method of the Reflected Scattered Waves. Japanese Journal of Applied Physics, 1996, 35(4): 3105-3108.

[96] H A Ocampo, Alfredo. Metodologia para la captura y analisis de ondas sismicas artificiales a partir de hologramas acusticos para la deteccion de objetos relacionados con minas anti persona. Colombia: Metropolitan Institute of Technology, 2014.

[97] L Li, J Tan, D A Wood, et al. A review of the current status of induced seismicity monitoring for hydraulic fracturing in unconventional tight oil and gas reservoirs. Fuel, 2019, 242: 195-210.

[98] W A Schneider. The common depth point stack. Proceedings of the IEEE, 1984, 72(10): 1238-1254

[99] C H Frazier, N Çadallı, J D C Munson, et al. Acoustic imaging of objects buried in soil. The Journal of the Acoustical Society of America, 2000, 108(1): 147-156

[100] Y Wei, X Shi, Q Liu, et al. The influence of crack modes on the elastic wave propagation characteristics in a non-uniform rotating shaft. Applied Sciences, 2018, 8(11): 2105.

[101] B Albers. Linear elastic wave propagation in unsaturated sands, silts, loams and clays. Transport in Porous Media, 2011, 86(2): 537-557.

[102] W C Lo, C L Yeh, CTTsai. Effect of soil texture on the propagation and attenuation of acoustic wave at unsaturated conditions. Journal of Hydrology, 2007, 338(3-4): 273-284.
[103] J M Muggleton, M J Brennan, C D F Rogers, et al. Point vibration measurements for the detection of shallow-buried objects. Tunnelling and Underground Space Technology, 2014, 39: 27-33.

[104] Y Liu, D Habibi, D Chai, et al. A comprehensive review of acoustic methods for locating underground pipeline. Applied Sciences, 2020, 10(3): 1031.

[105] J M Muggleton, E Rustighi. 'Mapping the Underworld': recent developments in vibro-acoustic techniques to locate buried infrastructure. Géotechnique Letters, 2013, 3(3): 137-141.

[106] P Zhang, L Zhang, TWu, et al. Detection and location of fouling on photovoltaic panels using a drone-mounted infrared thermography system. Journal of Applied Remote Sensing, 2017, 11(1): 016026.

[107] L Wang, Z P Wu, C Chen, et al. Using low altitude thermal infrared remote sensing technique to detect the underground oil pipelines. Science of Surveying and Mapping, 2018, 43(03): 142-147. (in Chinese)

[108] U Netzelmann, G Walle, S Lugin, et al. Induction thermography: principle, applications and first steps towards standardisation. Quantitative InfraRed Thermography Journal, 2016, 13(2): 170-181.

[109] G Shen, T Li. Infrared thermography for high-temperature pressure pipe. Insight-Non-Destructive Testing and Condition Monitoring, 2007, 49(3): 151-153.

[110] P M Bach, J K Kodikara. Reliability of infrared thermography in detecting leaks in buried water reticulation pipes. IEEE Journal of Selected Topics in Applied Earth Observations and Remote Sensing, 2017, 10(9): 4210-4224.

[111] M H Loke, J E Chambers, D F Rucker, et al. Recent developments in the direct-current geoelectrical imaging method. Journal of Applied Geophysics, 2013, 95: 135-156.

[112] A P Aizebeokhai. 2D and 3D geoelectrical resistivity imaging: Theory and field design. Scientific Research and Essays, 2010, 5(23): 3592-3605.

[113] E Attias, KWeitemeyer, S Hölz, et al. High-resolution resistivity imaging of marine gas hydrate structures by combined inversion of CSEM towed and ocean-bottom receiver data. Geophysical Journal International, 2018, 214(3): 1701-1714.

[114] C Ungureanu, A Priceputu, A L Bugea, et al. Use of electric resistivity tomography (ERT) for detecting underground voids on highly anthropized urban construction sites. Procedia Engineering, 2017, 209: 202-209.

[115] R Putiška, M Nikolaj, I Dostál, et al. Determination of cavities using electrical resistivity tomography. Contributions to Geophysics and Geodesy, 2012,42(2): 201-211.

[116] A Carollo, P Capizzi, R Martorana. Joint interpretation of seismic refraction tomography and electrical resistivity tomography by cluster analysis to detect buried cavities. Journal of Applied Geophysics, 2020: 104069.

[117] W Zhang, HY Xiao, Z C Zhong. Application of high density resistivity method to detecting urban underground water pipelines. Geotechnical Investigation \& Surveying, 2009, 37(07): 91-94. (in Chinese)

[118] S Shin, S Park, J H Kim. Time-lapse electrical resistivity tomography characterization for piping detection in earthen dam model of a sandbox. Journal of Applied Geophysics, 2019, 170: 103834.

[119] GWu, G Yang, H Tan. Mapping coalmine goaf using transient electromagnetic method and high density resistivity method in Ordos City, China. Geodesy and Geodynamics, 2016, 7(5): 340-347.

[120] KPhengnaone, R Arjwech, M Everett. 2D electrical resistivity tomography (ERT) method to delineate coal seams: Case studies on lignite and anthracite. Songklanakarin Journal of Science \& Technology, 2020, 42(4).

[121] K Shen, M S Selezneva, K A Neusypin. Development of an algorithm for correction of an inertial navigation system in Off-Line mode. Measurement Techniques, 2018, 60(10): 991-997.

[122] H Sahli. MEMS-based aided inertial navigation system for small diameter pipelines. Canada: University of Calgary, 2016

[123] Y Jia, S Li, Y Qin, et al. Error analysis and compensation of MEMS rotation modulation inertial navigation system. IEEE Sensors Journal, 2018, 18(5): 2023-2030.

[124] M S Chowdhury, M F Abdel-Hafez. Pipeline inspection gauge position estimation using inertial measurement unit, odometer, and a set of reference stations. ASCE-ASME Journal of Risk and Uncertainty in Engineering Systems, Part B: Mechanical Engineering, 2016, 2(2): 1-10.

[125] LW Guan, X D Cong, Q Zhang, et al. A comprehensive review of microinertial measurement unit based intelligent pig multi-sensor fusion 
technologies for small-diameter pipeline surveying. Micromachines, 2020, $11(9): 840$

[126] P L Hanna. Strapdown inertial systems for pipeline navigation. IEE Colloquium on Inertial Navigation Sensor Development. London, UK, Jan 9-9, 1990: 1-8.

[127] L Guan, Y Gao, H Liu, et al. A review on small-diameter pipeline inspection gauge localization techniques: Problems, methods and challenges. International Conference on Communications, Signal Processing, and their Applications (ICCSPA). Sharjah, United Arab Emirates, March 19-21, 2019: 1-6.

[128] D Sytnyk. Simulation environment for the unexmin underwater robotic exploration system. Institudo Superior de Engenharia do Porto, 2018.

[129] D Hyun, H S Yang, H S Park, et al. Dead-reckoning sensor system and tracking algorithm for 3-D pipeline mapping. Mechatronics, 2010, 20(2): 213-223.

[130] D H Lee, H Moon, J C Koo, et al. Map building method for urban gas pipelines based on landmark detection. International Journal of Control, 2013, 11(1): 127-135.

[131] Q Chen, Q Zhang, X Niu, et al. Positioning accuracy of a pipeline surveying system based on MEMS IMU and odometer: Case study. IEEE access, 2019, 7: 104453-104461.

[132] Q Chen, X Niu, J Kuang, et al. IMU mounting angle calibration for pipeline surveying apparatus. IEEE Transactions on Instrumentation and Measurement, 2019, 69(4): 1765-1774.

[133] W Abd-Elhamid, A Osman, A Noureldin, et al. Wavelet multi-resolution analysis for enhancing the performance of integrated GPS and MEMSbased navigation systems. Geomatica, 2005, 59(1): 61-72.

[134] W M F Al-Masri, M F Abdel-Hafez, M A Jaradat. Inertial navigation system of pipeline inspection gauge. IEEE Transactions on Control Systems Technology, 2018, 28(2): 609-616.

[135] Z Cui, Q Wang, Q Xue, et al. A review on image reconstruction algorithms for electrical capacitance/resistance tomography. Sensor Review, 2016, 36(4): 429-445.

[136] P N Darma, M R Baidillah, M W Sifuna, et al. Improvement of image reconstruction in electrical capacitance tomography (ECT) by sectorial sensitivity matrix using a K-means clustering algorithm. Measurement Science and Technology, 2019, 30(7): 075402.

[137] W N Abd Rashid, E J Mohamad, R A Rahim, et al. Design of nondestructive testing on composite material using parallel plate electrical capacitance tomography: a conceptual framework. Jurnal Teknologi, 2017, 79(5-2):1-6.

[138] B J Huber. Capacitive tomography for the location of plastic pipe. Gas Technology Institute (US), 2002:1-55.

[139] B J Huber. Capacitive tomography for the location of plastic pipe. US: Gas Technology Institute, 2002. https://www.osti.gov/biblio/809196

[140] G Guo, G Tong, L Lu, et al. Iterative reconstruction algorithm for the inverse problems in electrical capacitance tomography. Flow Measurement and Instrumentation, 2018, 64: 204-212.

[141] J Kryszyn, D M Wanta, WT Smolik. Gain adjustment for signal-to-noise ratio improvement in electrical capacitance tomography system EVT4. IEEE Sensors Journal, 2017, 17(24): 8107-8116.

[142] CTholin-Chittenden, M Soleimani. Planar array capacitive imaging sensor design optimization. IEEE Sensors Journal, 2017, 17(24): 8059-8071.

[143] Q Marashdeh, W Warsito, L S Fan, et al. Nonlinear forward problem solution for electrical capacitance tomography using feed-forward neural network. IEEE Sensors Journal, 2006, 6(2): 441-449.

[144] D J Pasadas, A L Ribeiro, H G Ramos, et al. Automatic parameter selection for Tikhonov regularization in ECT Inverse problem. Sensors and Actuators A: Physical, 2016, 246: 73-80.

[145] Z Cao, L Ji, L Xu. Iterative reconstruction algorithm for electrical capacitance tomography based on Calderon's method. IEEE Sensors Journal, 2018, 18(20): 8450-8462.

[146] B Inanloo, B Tansel, K Shams, et al. A decision aid GIS-based risk assessment and vulnerability analysis approach for transportation and pipeline networks. Safety Science, 2016, 84: 57-66.

[147] X Zhang, Y Han, D S Hao, et al. ARGIS-based outdoor underground pipeline information system. Journal of Visual Communication and Image Representation, 2016, 40: 779-790.

[148] N F Rezaei, M Karimi, G M Jabari. Qualitative risk assessment of gas pipelines by using of indexing system method in GIS environment.
Engineering Journal of Geospatial Information Technology, 2019, 7(1): 91-105.

[149] M Tulloch, M Chapman. Mapping Ontario's underground utilities. Ontario Prof Surv, 2005: 24-27.

[150] A Nasirian, M F Maghrebi, S Yazdani. Leakage detection in water distribution network based on a new heuristic genetic algorithm mode. Journal of Water Resource and Protection, 2013, 5(3): 294-303.

[151] A L Balogun, A N Matori, K Yussof, et al. GIS in pipeline route selection: current trend and challenges, 2013. http://mycoordinates.org/gis-in-pipel ine-route-selection/.

[152] A Agapiou, D D Alexakis, KThemistocleous, et al. Water leakage detection using remote sensing, field spectroscopy and GIS in semiarid areas of Cyprus. Urban Water Journal, 2016, 13(3): 221-231.

[153] S M Ghavami, Z Borzooei, J Maleki. An effective approach for assessing risk of failure in urban sewer pipelines using a combination of GIS and AHP-DEA. Process Safety and Environmental Protection, 2020, 133: 275-285.

[154] G Kennedy, V Ila, R Mahony. A perception pipeline for robotic harvesting of green asparagus. IFAC-PapersOnLine, 2019, 52(30): 288-293.

[155] W Zhao, M Kamezaki, K Yoshida, et al. A coordinated wheeled gas pipeline robot chain system based on visible light relay communication and illuminance assessment. Sensors, 2019, 19(10): 2322.

[156] J Feder. Smart robotic system tracks buried pipelines, inspects for external damage. Journal of Petroleum Technology, 2019, 71(12): 59-62.

[157] $\mathrm{H}$ Song, $\mathrm{K} \mathrm{Ge}, \mathrm{D}$ Qu, et al. Design of in-pipe robot based on inertial positioning and visual detection. Advances in Mechanical Engineering, 2016. https://doi.org/10.1177/1687814016667679.

[158] H Akbarzadeh, A Bayat. Investigating ASTM F 1962 arching factor for Polyethylene pipes installed by Horizontal Directional Drilling. Tunnelling and Underground Space Technology, 2014, 39: 50-57.

[159] T Zayed, M Mahmoud. Data acquisition and factors impacting productivity of Horizontal Directional Drilling (HDD). Tunnelling and underground Space Technology, 2013, 33: 63-72.

[160] S A Fjerdingen, P Liljebäck, A A Transeth. A snake-like robot for internal inspection of complex pipe structures (PIKo). 2009 IEEE/RSJ International Conference on Intelligent Robots and Systems, St. Louis, MO, USA, Oct. 10-15, 2009: 5665-5671.

[161] F Tâche, W Fischer, G Caprari, et al. Magnebike: A magnetic wheeled robot with high mobility for inspecting complex-shaped structures. Journal of Field Robotics, 2009, 26(5): 453-476.

[162] W Fischer, G Caprari, R Siegwart, et al. Foldable magnetic wheeled climbing robot for the inspection of gas turbines and similar environments with very narrow access holes. Industrial Robot: An International Journal, 2010, 37(3): 24-29.

[163] Q Tu, Q Liu, TRen, et al. Obstacle crossing and traction performance of active and passive screw pipeline robots. Journal of Mechanical Science and Technology, 2019, 33(5): 2417-2427.

[164] A Kakogawa, S Ma. Design of a multilink-articulated wheeled pipeline inspection robot using only passive elastic joints. Advanced Robotics, 2018, 32(1): 37-50.

[165] Y J Qiang, Z Chang, J H Chen. Research on detection method of buried gas PE pipeline in town. East China Cross-Strait Exchange Seminar, Taiwan, China, September 23, 2019: 26-28. (in Chinese)

[166] X R Zhou, H H Chen, J L Li. Mapping the buried pipelines from GPR and GPS data. 2nd International Conference on Software Engineering and Information Management, Changsha, China, December 6-7, 2019 : 199-203.

[167] C Q G Muñoz, F P G Marquez, B Lev, et al. New pipe notch detection and location method for short distances employing ultrasonic guided waves. Acta Acustica United with Acustica, 2017, 103(5): 772-781.

[168] R K Amineh, M Ravan, R Sharma. Nondestructive testing of nonmetallic pipes using wideband microwave measurements. IEEE Transactions on Microwave Theory and Techniques, 2020, 68(5): 1763-1772.

[169] HWu, M Ravan, R Sharma, et al. Microwave holographic imaging of non-metallic concentric pipes. IEEE Transactions on Instrumentation and Measurement, 2020: 1-13.

[170] Q Ma, GTian, Y Zeng, et al. Pipeline in-line inspection method, instrumentation and data management. Sensors, 2021, 21(11): 3862. https:// doi.org/https://doi.org/10.3390/s21113862. 